1

15 Psychology, School of Social Sciences

16 Nanyang Technological University

$17 \quad$ Singapore 639818

18 Phone: +65 6592-1571

19 Fax: +65 6795-5797

20 Email: xuhong@ntu.edu.sg

Declarations of interest: none

*Correspondence:

Dr. Hong Xu

48 Nanyang Ave, HSS-04-06

\author{
Email:xuhong@ntu.edu.sg
}

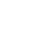

7 Abstract word count: 312

Introduction and Discussion word count: 2990

Method and Results word count: 5623

Number of References: 76 \title{
mechanisms \\ Temporal and spatial ensemble statistics are formed by distinct
}

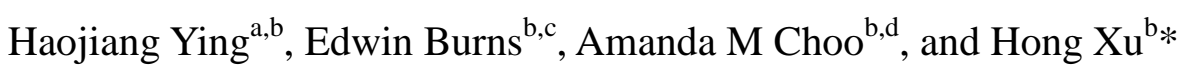
${ }^{a}$ Department of Psychology, Soochow University, Suzhou, China ${ }^{c}$ Department of Psychology, University of Richmond, USA

${ }^{\mathrm{d}}$ School of Biological Sciences, Nanyang Technological University, Singapore

Manuscript accepted for publication in Cognition on the 04/11/2019

${ }^{\mathrm{b}}$ Psychology, School of Social Sciences, Nanyang Technological University, Singapore 


\section{Abstract}

Our brains can extract a summary representation of the facial characteristics provided by a group of faces. To date, there has been a lack of clarity as to what calculations the brain is actually performing during this ensemble perception. For example, does ensemble processing average the fiducial points (e.g., distance between the eyes, width of the mouth) and surface characteristics (e.g., skin tone) of a set of faces in a fashion that produces what we call a 'morph average' face from the group? Or does ensemble perception extract a general 'gist average' of the face set (e.g., these faces are unattractive)? Here, we take advantage of the fact that the 'morph average' face derived from a group of faces is more attractive than the 'gist average'. If ensemble perception is performing morph averaging, then the adaptation aftereffects elicited by a morphed average face from a group should be equivalent to those elicited by the group. By contrast, if ensemble perception reflects gist averaging, then the aftereffects produced by the group should be distinct from those elicited by the more attractive morphed average face. In support of the morph averaging hypothesis, we show that the adaptation aftereffects derived via temporal ensemble perception of a group of faces are equal to those produced by the group's morphed average face. Moreover, these effects increase as a linear function of increasing attractiveness in the underlying group. We also reveal that spatial ensemble processing is not equal to temporal ensemble processing, but instead reflects the 'gist' attractiveness of the group of faces; e.g., these faces are unattractive. Finally, we show that gist averaging of a spatially presented group of faces is abolished when a temporal manipulation is additionally employed; under these circumstances, morph averaging becomes apparent again. In summary, we have shown for the first time that temporal and spatial ensemble statistics reflect qualitatively different perceptual calculations. Keywords: rapid serial visual presentation, adaptation, ensemble statistics, face, attractiveness 


\section{Introduction}

When we are presented with an array of stimuli in a scene, our brains involuntarily extract the ensemble statistics of the information that they convey (Alvarez, 2011; Haberman \&

27 Whitney, 2007, 2012; Whitney \& Yamanashi Leib, 2017). For example, we can accurately report 28 the mean emotion from a group of emotional faces (Haberman \& Whitney, 2007, 2009; Whitney

29 \& Yamanashi Leib, 2017; Wolfe, Kosovicheva, Leib, Wood, \& Whitney, 2015; Ying \& Xu, 2017).

30 Such averaging is considered to be a type of ensemble statistics (Alvarez, 2011; Ariely, 2001;

31 Haberman, Brady, \& Alvarez, 2015; Haberman \& Whitney, 2007, 2009, 2012; Whitney \&

32 Yamanashi Leib, 2017; Ying \& Xu, 2017), and can occur both spatially (i.e., multiple faces

33 presented at once in a scene; e.g., Haberman \& Whitney, 2007, 2009; Ying, Burns, Lin, \& Xu,

34 2019) and temporally (i.e., different faces presented one at a time in rapid succession; e.g.,

35 Haberman, Harp, \& Whitney 2009; Ying \& Xu, 2017).

Despite researchers widely describing ensemble statistics as extracting the gist of a scene,

37 it is still far from clear what this 'gist' represents (Alvarez, 2011; Whitney \& Yamanashi Leib,

38 2017). For example, does ensemble coding extract a general representation of the group's mean

39 characteristics, whereby the faces are summarized via what we call 'gist averaging'; e.g., the

40 mean attractiveness of these unattractive faces is unattractive? Alternatively, does the brain

41 calculate the fiducial points for each face (e.g., distance between eyes, width of the lips) with

42 their surface characteristics (e.g., the redness of the cheeks), and then average them together to

43 create a new mean face derived from this information? We call this latter form of ensemble

44 coding 'morph averaging' due to the fact that it is very similar to how specialist computer

45 morphing software creates an average face from a group of faces (Debruine \& Tiddeman, 2017,

46 Tiddeman, Burt, \& Perrett, 2001). 
Remarkably to date, there has been no clear evidence to support either the gist or morph averaging accounts of ensemble coding. Here, we tested these potential hypotheses by taking advantage of the well-established fact that a computer-generated average face, created by averaging the fiducial points and surface characteristics of a group of faces, is generally more attractive than the individual faces from which the average is comprised (DeBruine, Jones, Unger,

52 \& Little, 2007; Galton, 1878; Perrett, May, \& Yoshikawa, 1994; Valentine, Darling, \& Donnelly,

53 2004). This effect has been documented from the dawn of modern psychology, with Galton

54 (1878) relaying that averaging leads to '....in every instance, a decided improvement of beauty'

55 (Valentine et al., 2004). By requiring participants to perceive facial attractiveness in a temporal ensemble fashion, we can clearly test for the first time whether the morph average (i.e., the ensemble statistics of the group is equivalent to the morphed average face, such that a group of unattractive faces should no longer be perceived as unattractive) or the gist average (i.e., ensemble perception of the group should be less attractive than the morph average, such that a group of unattractive faces remains unattractive) hypothesis of ensemble coding is correct.

We therefore adapted participants to a group of faces presented one at a time in rapid serial visual presentation (RSVP; Potter, 1976). We chose an adaptation paradigm instead of a

63 direct rating approach as adaptation is a powerful method that can detect perceptual effects even

64 when explicit ratings are unable to (Liu, Montaser-Kouhsari, \& Xu, 2014). After adapting to a

65 face for a few seconds, the facial characteristics of the adapting face appear less apparent in

66 subsequently viewed faces (Leopold, O'Toole, Vetter, \& Blanz, 2001; Luo, Burns, \& Xu, 2017 ;

67 Rhodes \& Jeffery, 2006; Webster, Kaping, Mizokami, \& Duhamel, 2004; Webster \& MacLeod, 68 2011; Xu, Dayan, Lipkin, \& Qian, 2008; Ying \& Xu, 2017); thus, adapting to an attractive face 69 will lead to the subsequently viewed face as being less attractive; a powerful visual illusion 
70 known as an attractiveness adaptation aftereffect (Pegors, Mattar, Bryan, \& Epstein, 2015;

71 Rhodes, Jeffery, Watson, Clifford, \& Nakayama, 2003; Ying et al., 2019). The magnitudes of

72 these adaptation aftereffects reflect the strength of different attributes present in the adapting

73 face; i.e., an extremely attractive face will produce larger aftereffects than a face that is only

74 moderately attractive (e.g., Webster et al., 2004; Ying et al., 2019). In our first experiment, we

75 therefore compared the adaptation aftereffects produced by a group of RSVP faces, versus those

76 elicited by their computer-generated, morph average: if they are indistinguishable from one

77 another, then it would suggest that ensemble statistics is not a simple extraction of the group's

78 gist (e.g., these faces are unattractive), but instead stems from a process that is consistent with

79 morph averaging the fiducial points and surface aspects of the faces together. By contrast, if our

80 gist averaging hypothesis is correct, the computer-generated morph average face should produce

81 adaptation aftereffects that are distinct from the RSVP streams. This is because the computer-

82 generated morph average face is invariably more attractive than the underlying group it is

83 comprised of (DeBruine et al., 2007; Perrett et al., 1994; Valentine et al., 2004).

\section{Experiment 1: Temporal ensemble statistics represent morph averaging}

In our first experiment, we directly tested our morph versus gist averaging hypotheses by

87 comparing the adaptation aftereffects produced by an RSVP stream of faces to the morphed

88 average face derived from their group. If ensemble coding represents the morph average, then we

89 should observe (a) similar and correlated aftereffects between the RSVP face stream and its

90 computer-generated morph average, and (b) since this morph average will be more attractive

91 than the individual faces in the group, the unattractive face stream may fail to generate

92 aftereffects in the direction that we would expect from those typically induced by unattractive 
93 faces (e.g., the faces may produce no aftereffects, or even make faces presented after them seem

94 less attractive). On the other hand, if the ensemble coding represents gist averaging, then the

95 unattractive face stream should generate a significant aftereffect (e.g., faces presented after the

96 stream should appear more attractive relative to no adaptation baseline) since the gist average of

97 an unattractive face stream is still considered to be unattractive.

\section{Experiment 1: Methods}

\section{Participants}

Twenty-nine participants (14 Females; Mean Age: 22.03) with normal or corrected-to-

102 normal vision were recruited from Nanyang Technological University. We aimed to recruit 30

103 participants; however, one dropped out during the experiment and was not replaced, thus leaving

104 us with only 29 participants. We selected this sample size based upon previous face 105 attractiveness adaptation work ( $n=30$ in Pegors et al, 2015). Written informed consent was 106 provided by participants in all four experiments beforehand. This study was approved by the 107 Institutional Review Board (IRB) at Nanyang Technological University, Singapore, in 108 accordance with the Code of Ethics of the World Medical Association (Declaration of Helsinki) 109 for experiments involving human participants.

\section{Apparatus}

112 Visual stimuli were presented on a 17-inch Philips CRT monitor (refresh rate $85 \mathrm{~Hz}$,

113 spatial resolution $1024 \times 768$ pixels; comparison between CRT and LCD monitor can be found 114 in Zhang et al., 2018). The monitor was controlled by an iMac Intel Core i3 computer running 
115 Matlab R2010a (Mathworks, MA, USA) via Psychophysics Toolbox (Brainard, 1997; Pelli,

116 1997). The experiment was conducted in a dimly lit room. During the experiment, participants

117 rested their heads on a chin rest $75 \mathrm{~cm}$ in front of the monitor. Each pixel subtended $0.024^{\circ}$ on

118 the screen.

Visual Stimuli

Thirty-Five Chinese female faces were chosen from the N-FEE database (Yap, Chan \&

122 Christopoulos, 2016). Due to copyright restrictions we are not allowed to publicly publish these

123 images, so we have used faces from the KDEF database for illustrative purposes (Lundqvist,

124 Flykt, \& Öhman, 1998). In this study, we only selected portrait pictures from 35 female Chinese

125 Singaporeans with neutral expressions. All face images were grey scaled and masked so that only

126 the facial region of each face was visible to the participants. The luminance of the face images

127 was equalized via SHINE toolbox (Willenbockel et al., 2010). Every participant rated the

128 attractiveness of the 35 faces at least two weeks before the main experiment in Experiment 1

129 (adapted from Rhodes \& Jeffery, 2006; 1 for most unattractive and 7 for most attractive). Prior to

130 rating, participants were exposed to all of the faces, each for $400 \mathrm{~ms}$ in a randomized order, in

131 order to gauge the range of attractiveness in the faces before rating each face. Each face was

132 rated four times, with the mean rating for each face ranging between 2.67 and $5.00(M=3.53$,

$133 S D=1.31)$. Inter-rater reliability was high (Cronbach's alpha $=.98)$. The adapting stimuli were

134 selected from the four faces rated as most attractive and the four that were least attractive.

135 The test faces included one of the most attractive and one of the most unattractive faces

136 from the originally rated 35 faces (excluding the adaptors), and a further five faces that were

137 produced by morphing these two faces in equally incremental steps between them (thus giving us 
1387 attractiveness units ranging from the original unattractive face through to the original attractive

139 face) via Webmorph (Debruine \& Tiddeman, 2017). Therefore, there are seven test faces in total.

140 To minimize low-level adaptation as per prior research (Burns et al., 2017; Rhodes et al., 2003;

141 Ying \& Xu, 2017; Zhao \& Chubb, 2001), the adapting stimuli were displayed at $3.20^{\circ} \times 4.03^{\circ}$,

142 which was roughly $133 \%$ of the size of the test stimuli. The adapting stimuli and the test stimuli

143 were always presented at the same side of the central fixation cross within one trial, and their

144 centers were roughly $3.8^{\circ}$ away from the central fixation cross (159 pixels). Our reason for

145 presenting the faces in the periphery was because adaptation aftereffects have been found to be

146 greater in the visual periphery compared to the fovea (Bachy \& Zaidi, 2014; Chen, Chen, Gao,

147 Yang, \& Yan, 2015; Ying \& Xu, 2017). Similar to Haberman, Lee, and Whitney (2015), we are

148 aware that the 'attractiveness unit' is arbitrary, and we do not mean that the (perceived)

149 attractiveness differences between the testing faces are strictly linear. The 'attractiveness unit'

150 merely represents the relative differences between these faces.

\section{Procedure}

Participants completed five blocks: baseline, RSVP unattractive, RSVP attractive,

154 computer-generated average unattractive morph, and computer-generated average attractive

155 morph. In the baseline condition, participants simply rated the test faces, which were presented

156 for $400 \mathrm{~ms}$, as attractive or unattractive. Each test face was presented 10 times at random giving

157 a total of 70 trials in each block. The same test face sampling occurred in the attractive RSVP

158 block, but this time participants viewed an RSVP stream of the four attractive adapting faces

159 prior to viewing each test face. The temporal frequency of the RSVP sequence was $42.5 \mathrm{~Hz}$, with

160 each face displayed for $23.5 \mathrm{~ms}$ per face frame (with no interval between two face frames, the 
161 same as Ying \& $\mathrm{Xu}, 2017)$. Thus, each adapting face was presented 40 times, in a random order,

162 during the $3.764 \mathrm{~s}$ adaptation phase $(23.5 \mathrm{~ms} \times 4$ faces $\times 40$ repetitions $)$. Figure 1 displays the

163 trial sequence. This method was repeated for the unattractive RSVP block, except the RSVP

164 stream comprised the unattractive adaptors. The same process occurred for the attractive

165 morphed average block, except during adaptation when participants were simply presented with

166 a single face that was created by morphing all of the four attractive adaptors' visual properties

167 together. The same was true for the unattractive morphed average block, except the unattractive

168 adaptors were used to create its adapting face morph. The blocks were presented in a random

169 order, with instructions given beforehand. Participants were given breaks that were roughly equal

170 in duration to an experimental block to disperse any carryover effects. Participants practiced for

$1715-10$ trials before participating in each of the experiments reported here.

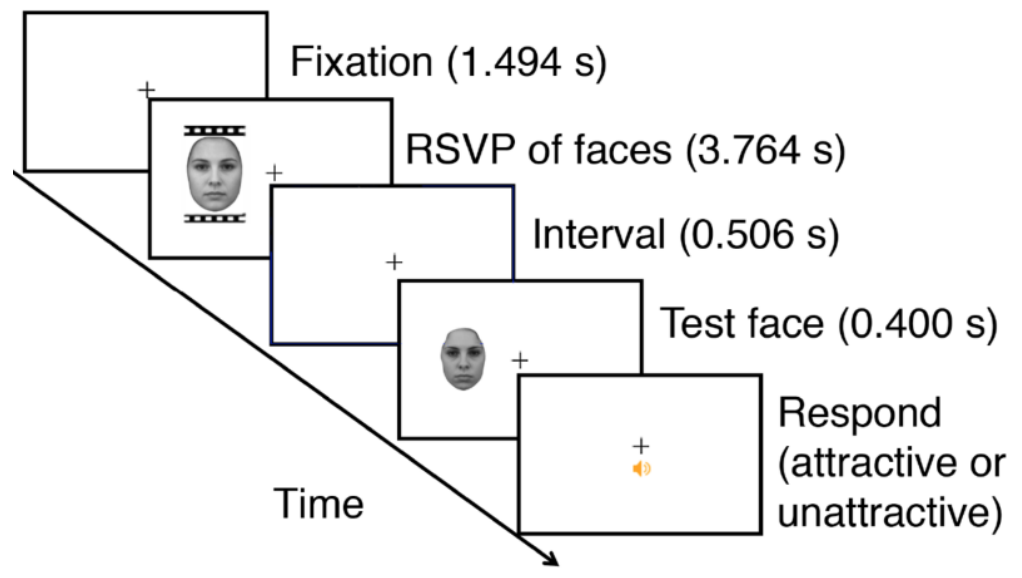

Figure 1. Example trial sequence from the RSVP adaptation condition (the demonstrated faces are

174 AF01NES and AF34NES from the KDEF database). Participants fixated on the cross at all times. After $1.494 \mathrm{~s}$, the 175 RSVP of the faces appeared onscreen for $3.764 \mathrm{~s}$. After a short interval $(0.506 \mathrm{~s})$, the test face appeared for $0.4 \mathrm{~s}$.

176 Then a beep sound prompted participants to judge the target face by pressing the 'A' button as attractive, or the ' $\mathrm{S}$ ' 177 button as unattractive.

In each trial, the test stimulus presented was one of the seven test faces selected at

180 random. After that, a $50 \mathrm{~ms}$ beep sound prompted for participants to respond. Participants had to 
181 press the "A" or "S" key to express whether they found the test faces "attractive" or "unattractive"

182 respectively. Such two-alternative forced choice (2-AFC) methods have been commonly used in

183 adaptation experiments (e.g., Fox \& Barton, 2007; Webster et al., 2004; Xu, Dayan, Lipkin, \&

184 Qian, 2008). After the participant responded in each trial, the trial would terminate, thus

185 commencing the next trial. No feedback was given throughout. Within each block there were 70

186 trials, which comprised a presentation of each of the 7 test faces 10 times in a random sequence.

Analysis

Participants' responses were sorted into proportions of 'attractive' responses to each test

190 stimulus per adaptation condition. A psychometric curve was created with the $\mathrm{x}$-axis indexing the

191 test stimuli and the y-axis plotting the fractions of 'attractive' responses. Subsequently, the

192 psychometric curves were fitted with a sigmoidal function $\left.f(x)=1 /\left[1+e^{-a(x-b}\right)\right]$, where $a / 4$ is the

193 slope and $b$ provides the test-stimulus parameter corresponding to $50 \%$ of the psychometric

194 function, the point of subjective equality (PSE). We measured the adaptation aftereffects by

195 comparing the difference between the PSEs of the adapting conditions and the baseline condition.

196 Any subsidiary pairwise comparisons after the analysis of variance (ANOVA) were Bonferroni

197 corrected. Note that goodness of fit was evaluated by coefficient of determination $\left(\mathrm{R}^{2}=1\right.$

198 indicates the perfect fit). The mean goodness of fit $\left(\mathrm{R}^{2}\right)$ for all experiments was $>0.89$,

199 indicating that the predicted lines fitted the observed data well.

200 To confirm that any non-significant results truly supported the null hypothesis, we used

201 Bayes Factors to analyze the data (Dienes, 2014; Rouder, Speckman, Sun, Morey, \& Iverson, 202 2009) in addition to the traditional Frequentist analyses. In brief, Bayes Factor utilizes the 203 observed evidence for either the null or alternative hypothesis, with this weight of evidence 
204 realized as a ratio between the likelihoods of the hypotheses. For instance, ' $B F_{01}=3$ ' suggests

205 that the observed data is 3 times more likely to fit the null-hypothesis compared to the alternative

206 hypothesis. Generally, $B F_{01}>3$ is suggested to provide evidence for the null hypothesis. All

207 statistical analyses were conducted in JASP 0.8.6 (JASP team, 2018), R 3.4.3 (R Core Team,

208 Vienna, Austria), Matlab R2017a (Mathworks, MA, USA) and SPSS Statistics 22 (IBM, NY,

209 USA).

\section{Experiment 1: Results and Discussion}

The results from all the participants judging the facial attractiveness of the test faces

213 under various conditions are shown in Figure 2A. We plotted the fraction of attractive responses

214 as a function of the proportion of attractiveness of the test faces. The black (solid line with filled

215 squares) psychometric curve is the baseline condition without adaptation. After adapting to the

216 most attractive RSVP face stream, the participants judged the test faces as unattractive more

217 frequently than baseline, and the psychometric curve (blue dashed line with open diamonds,

$218 \mathrm{RSVPa}$ ) shifted to the right. This is the standard facial-attractiveness aftereffect (Hsu \& Young,

219 2004; Webster et al., 2004). The same finding occurred after adapting to the morphed average of

220 this face stream (light blue solid line with filled diamonds, Statica). Curiously, after adapting to

221 the most unattractive face stream (red dotted line with circles, RSPVu) or its morphed average

222 (magenta dashed-dotted line with filled circles, Staticu), there were no adaptation aftereffects

223 observed relative to baseline.

To determine the presence of adaptation aftereffects in our experiment, we performed 225 paired $t$-tests between the baseline PSE and the PSEs of the adaptation conditions (Figure 2B). 
As expected, both the attractive RSVP and morph average conditions produced significant

227 aftereffects (both $p s<.001$ ), with participants reporting the test faces as unattractive more

228 frequently in the two attractive conditions relative to the no adaptation baseline. Surprisingly,

229 neither of the unattractive conditions produced any aftereffects (both $p s>.62$ ). Bayesian $t$-tests

230 provided further support for the null hypothesis (RSVPu: $B F_{01}=4.52$; Staticu: $\left.B F_{01}=5.06\right)$ : the

231 unattractive conditions did not generate significant aftereffects relative to baseline. Participants

232 did not seem to be processing either set of unattractive adaptors as unattractive. These findings

233 contradict the outcome predicted by the gist averaging hypothesis, for if this hypothesis had been

234 correct, then the unattractive RSVP group should have displayed aftereffects that shifted the

235 psychometric curve in the opposite direction to those found in our attractive conditions (i.e.,

236 negative relative to baseline, where test faces were rated as attractive more frequently after

237 adaptation).

To test whether temporal ensemble perception was indistinguishable from the computer-

239 generated morph average, we performed a two-way repeated-measures ANOVA on the PSE

240 shifts relative to baseline with factors of Attractiveness (attractive vs. unattractive) and Adaptor

241 (RSVP vs. morph average). While there was a significant main effect of Attractiveness $(F(1,28)$

$\left.242=49.55, p<.001, \eta_{\mathrm{p}}{ }^{2}=.64\right)$ due to the attractive conditions producing larger aftereffects than the

243 unattractive conditions, there was no significant main effect of Adaptor $(F(1,28)=0.001, p=.99$,

$\left.244 \eta_{\mathrm{p}}^{2}<.001\right)$ nor any interaction $\left(F(1,28)=0.46, p=.50, \eta_{\mathrm{p}}^{2}=.016\right)$. Bayesian $t$-tests comparing

245 the attractive RSVP condition versus the attractive morph average $\left(B F_{01}=4.57\right)$, and the

246 unattractive RSVP versus the unattractive morph average $\left(B F_{01}=4.50\right)$, provided further

247 evidence for the null hypothesis. This confirms that the RSVP streams were processed by our

248 participants in a similar way to their morph averages. Further support for this came from the fact 
that the aftereffects from attractive $(r=.65, p<.001$; blue open diamonds with dashed line in

250 Figure 2C) and unattractive ( $r=.43, p=.019$; red full circles with solid line) RSVP streams were

251 correlated with their computer-generated morphed average face counterparts.

A

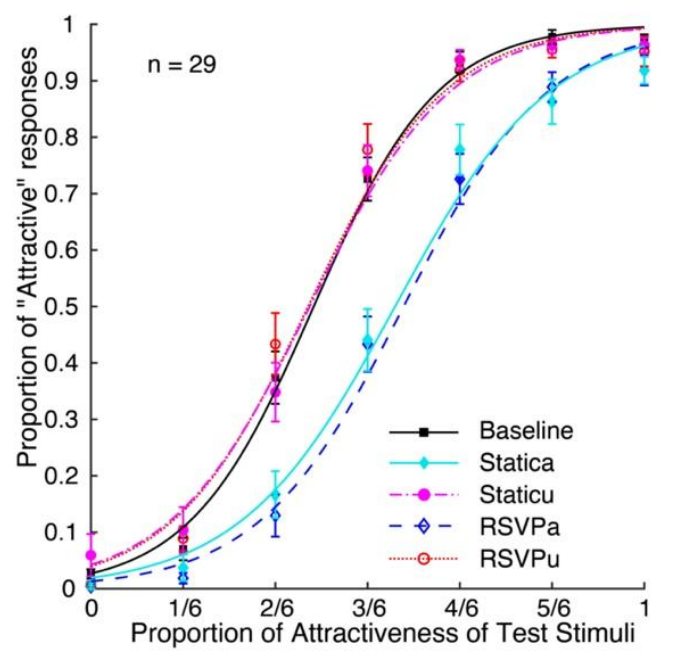

B

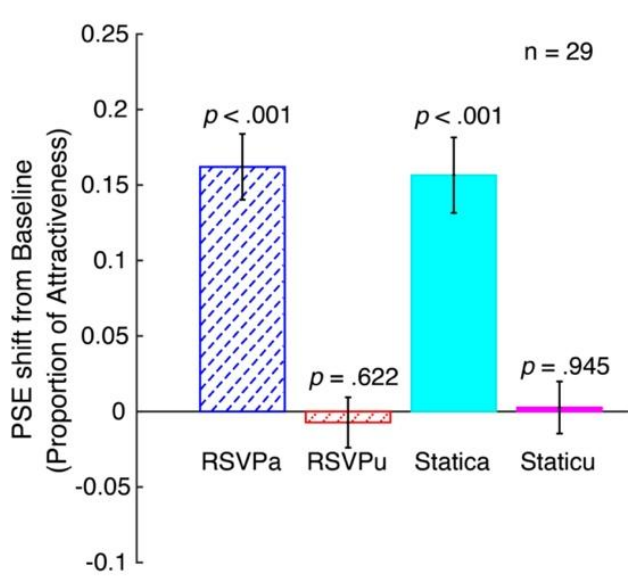

C

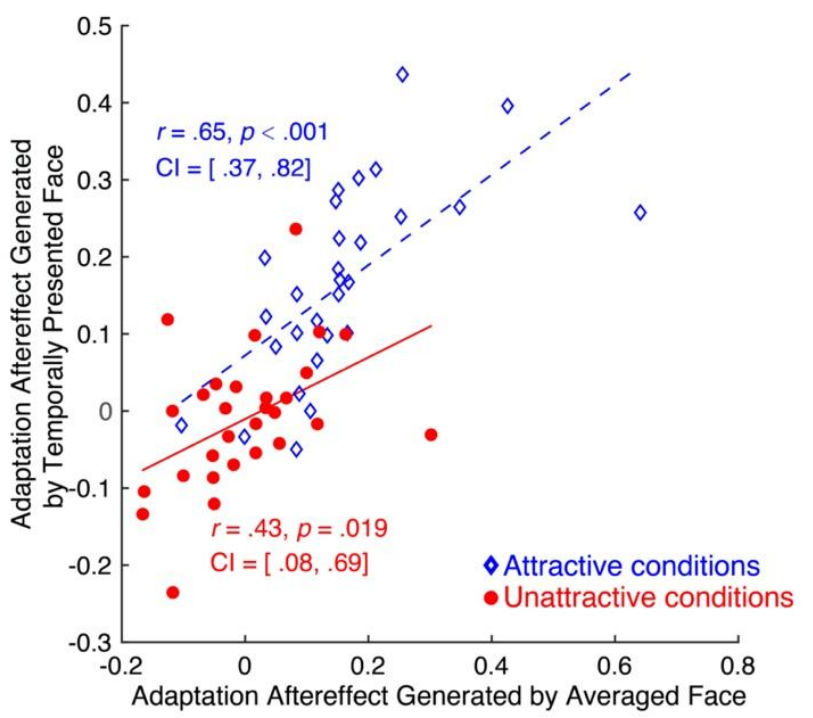

Figure 2. The RSVP and computer-generated morph average aftereffects (Experiment 1). (A) The psychometric functions of all participants averaged together. Error bars indicate the standard error of the mean. (B) Summary of all participants' results. For each condition, the adaptation aftereffect measured by PSE shift relative to baseline and the SEMs were plotted. The $p$-value shown for each condition in the figure was calculated using paired $t$-tests. Noticeably, a positive adaptation aftereffect measured by PSE shift indicates the target faces were perceived as less attractive than during baseline. The following figures adopt the same statistical analyses. (C) The relationship between the RSVP conditions and the paired morph average conditions. Each dot represents data from one participant: blue open diamond for the attractive conditions, and red filled circle for the unattractive conditions. 
263 We found that adapting to an RSVP stream and its computer-generated morphed average face led 264 to comparable, and correlated, facial attractiveness aftereffects. While these findings replicate 265 prior work that showed similar effects for facial emotion (Ying \& Xu, 2017), our results clarify 266 what characteristics of a face are extracted in order to produce temporal ensemble perception.

267 For example, the lack of differences between the morphed average faces and their RSVP groups 268 suggest that morph, rather than gist, averaging occurs during temporal ensemble coding. If gist 269 averaging had been occurring, then adapting to the unattractive face stream should have induced

270 aftereffects where the viewer rated subsequently presented test faces as attractive more often than 271 in the baseline. We did not observe this effect here with our unattractive RSVP group, instead, 272 these faces produced no aftereffects, with aftereffects actually comparable to their morphed 273 average counterpart. However, we do not think that this finding indicates that these faces were 274 not processed at all during adaptation. We believe that the data simply fits with the hypothesis 275 that the participants were morph averaging these faces together so that the group of unattractive 276 faces were processed as more attractive (i.e., roughly equal to baseline levels) than what they 277 were (i.e., unattractive). A similar lack of differences was found between the aftereffects 278 produced by the attractive group and its morphed average face. To our knowledge, this is the first 279 time that the morph averaging hypothesis of ensemble perception has been demonstrated as 280 having empirical support over the gist hypothesis. 


\section{Experiment 2: Temporal ensemble coding is driven by the underlying}

\section{mean attractiveness of the group}

In Experiment 1, adapting to unattractive RSVP faces produced no significant adaptation

aftereffects. We do not believe that this was due our participants not processing the unattractive the gist attractiveness of the individual faces in the group (i.e., unattractive). If this is the case, then adding in a new mixed ('MIX') condition, comprised of attractive and unattractive faces,

290 should induce aftereffects somewhere in between those observed for the attractive and

291 unattractive conditions in Experiment 1. Moreover, the magnitudes of these aftereffects across all

292 conditions should also be associated with the underlying mean attractiveness of the individual

293 faces, thereby demonstrating that our visual system adapts to the RSVP of face streams in a

294 linear fashion that is consistent with the principles of ensemble coding.

\section{Experiment 2: Methods}

Twenty new participants (10 Females; Mean Age: 22.84) participated in this experiment.

298 We selected this sample size for two reasons: firstly, a power analysis based upon the effect size

299 of Experiment $1\left(\eta_{\mathrm{p}}{ }^{2}=.65\right.$; using $\mathrm{G}^{*}$ Power 3.1 software; Faul, Erdfelder, Buchner, \& Lang,

300 2009), with $\alpha$-value at .05 , and power $(1-\beta)$ at .80 indicated that we needed at least 7

301 participants. However, considering the differences in experimental design, we chose to greatly

302 expand this number to roughly triple that sample size.

We used the same adaptation procedure as in Experiment 1, except there were three

304 adaptation conditions in addition to the baseline: RSVP of attractive faces ('ATT', four attractive 
faces), RSVP of mixed faces ('MIX', four attractive faces and four unattractive faces), and RSVP

306 of unattractive faces ('UNA', four unattractive faces) at a reduced adaptation duration (1.88 s in

307 Exp 2 vs. $3.764 \mathrm{~s}$ in Exp1). Note that in the 'MIX' condition the adapting RSVP streams were

308 presented for the same duration as the 'ATT' and 'UNA' conditions (see Experiment 1, Methods

309 section). Thus, in the 'MIX' condition, each adapting face was only presented 10 times during

310 the adaptation phase, so that the adapting duration is equated across different conditions. Also,

311 each test face in each block appeared 12 times in a random order. Additionally, after the main

312 experiment, we asked the participants to rate the mean attractiveness of the RSVP sequences on a

313 7-point scale (1 for most unattractive and 7 for most attractive), with each stream presented 10

314 times. These RSVP sequences were randomly presented for the same duration $(42.5 \mathrm{~Hz} ; 80$

315 frames $\times 23.5 \mathrm{~ms}$; in total $1.88 \mathrm{~s}$ ) as that during the adapting stage in the main experiment.

316 Since our data consisted of repeated measures from three observations (i.e., an

317 observation from each of the unattractive, mixed, and attractive conditions) for each participant,

318 we used the repeated measures correlation analysis (Bakdash \& Marusich, 2017) to quantify the

319 strength of the relationship between the attractiveness ratings of the faces and the adaptation

320 aftereffects produced by those faces. It uses the analysis of covariance (ANCOVA) to

321 'statistically adjust for inter-participant variability', thus 'estimates the common regression slope'

322 (generating the same slope), in other words, the association shared among individuals.

\section{Experiment 2: Results and Discussion}

The mean adaptation results from all participants are shown in Figure 6A. Similar to

326 Experiment 1, the RSVP of the Attractive condition generated a significant rightward shift of the 
327 psychometric curve, while the RSVP of the Unattractive condition failed to produce a shift.

328 Interestingly, the RSVP of the Mixed condition generated a smaller yet substantial rightward 329 shift. Relative to baseline, significant aftereffects were generated by the RSVPs of attractive

330 (Figure $3 \mathrm{~A}, M=.22, S E M=.004 ; t(19)=5.85, p<.001)$ and $\operatorname{mixed}(M=.12, S E M=.002 ; t(19)$

$331=5.06, p<.001)$ but not the unattractive $(M=.01, S E M=.02 ; t(19)=.47, p=.64)$ faces.

332 Bayesian analyses suggested that the lack of aftereffects in the unattractive condition was in 333 favor of the null hypothesis $\left(B F_{01}=3.89\right)$; i.e., no adaptation aftereffect relative to baseline.

334 Participants therefore rated the test faces as less attractive after adapting to the attractive and 335 mixed RSVP streams (Figure 3C). Moreover, we replicated Experiment 1 in showing no 336 aftereffects in the unattractive group, suggesting participants were not processing the RSVP 337 stream as unattractive. An ANOVA yielded significant differences among all three adaptation 338 conditions (with Greenhouse-Geisser correction, $F(1.55,29.36)=33.22, p<.001, \eta_{\mathrm{p}}{ }^{2}=.64$ ).

339 Subsidiary Bonferroni corrected comparisons showed significant differences between the 340 attractive and unattractive $(t(19)=6.73, p<.001)$, attractive and mixed $(t(19)=3.88, p=.003)$,

341 and mixed and unattractive $(t(19)=5.86, p<.001)$ conditions.

343 'Unattractive' conditions, it should in theory yield an aftereffect which is roughly equal to the

344 mean of those of two conditions. We therefore compared the adaptation aftereffects of the 'MIX'

345 condition with the average of the aftereffects from those two conditions. The paired samples $t$ -

346 test suggested that there was no significant difference between this pair $(t(19)=.28, p=.78$,

$\left.347 B F_{01}=4.15\right)$. Therefore, the 'Mixed' condition closely resembles the midpoint of the 'Attractive'

348 and 'Unattractive' conditions. This indicates that the participants perceived the attractiveness of

349 the adapting stream in a graded fashion consistent with ensemble coding. 
An ANOVA on the participants' attractiveness ratings of the RSVP streams showed they

351 were also significantly different from one another $\left(F(2,38)=112.55, p<.001, \eta_{\mathrm{p}}^{2}=.86\right)$.

352 Further comparisons indicated that participants judged the RSVP of the attractive faces $(M=$

$3534.89, S E M=.013)$ as the most attractive, followed by the RSVP of mixed faces $(M=3.98, S E M$

$354=.016)$, and the RSVP of unattractive faces $(M=2.65, S E M=.017)$ were judged as least

355 attractive (all $p s<.001$ ). Further repeated measures correlation analyses (Bakdash \& Marusich, 356 2017) revealed a significant positive correlation between the attractiveness ratings of the RSVP

357 streams and the adaptation aftereffects (Figure 3D, $r=.71, p<.001,95 \%$ CI $[0.50,0.84]$ );

358 indicating that the brain performs temporal ensemble statistics in a linear fashion from the 359 underlying attractiveness of the stream. 
A

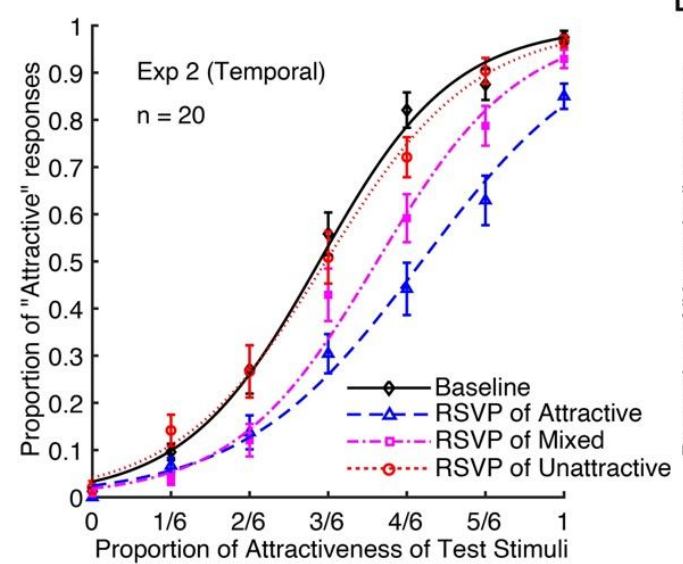

B

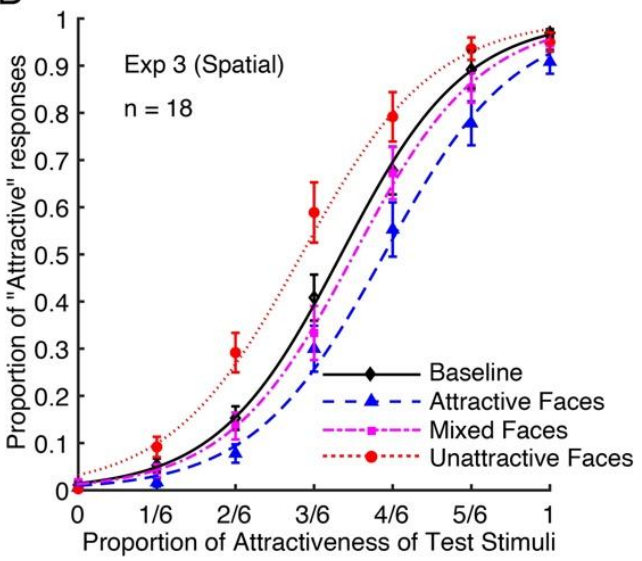

C

C
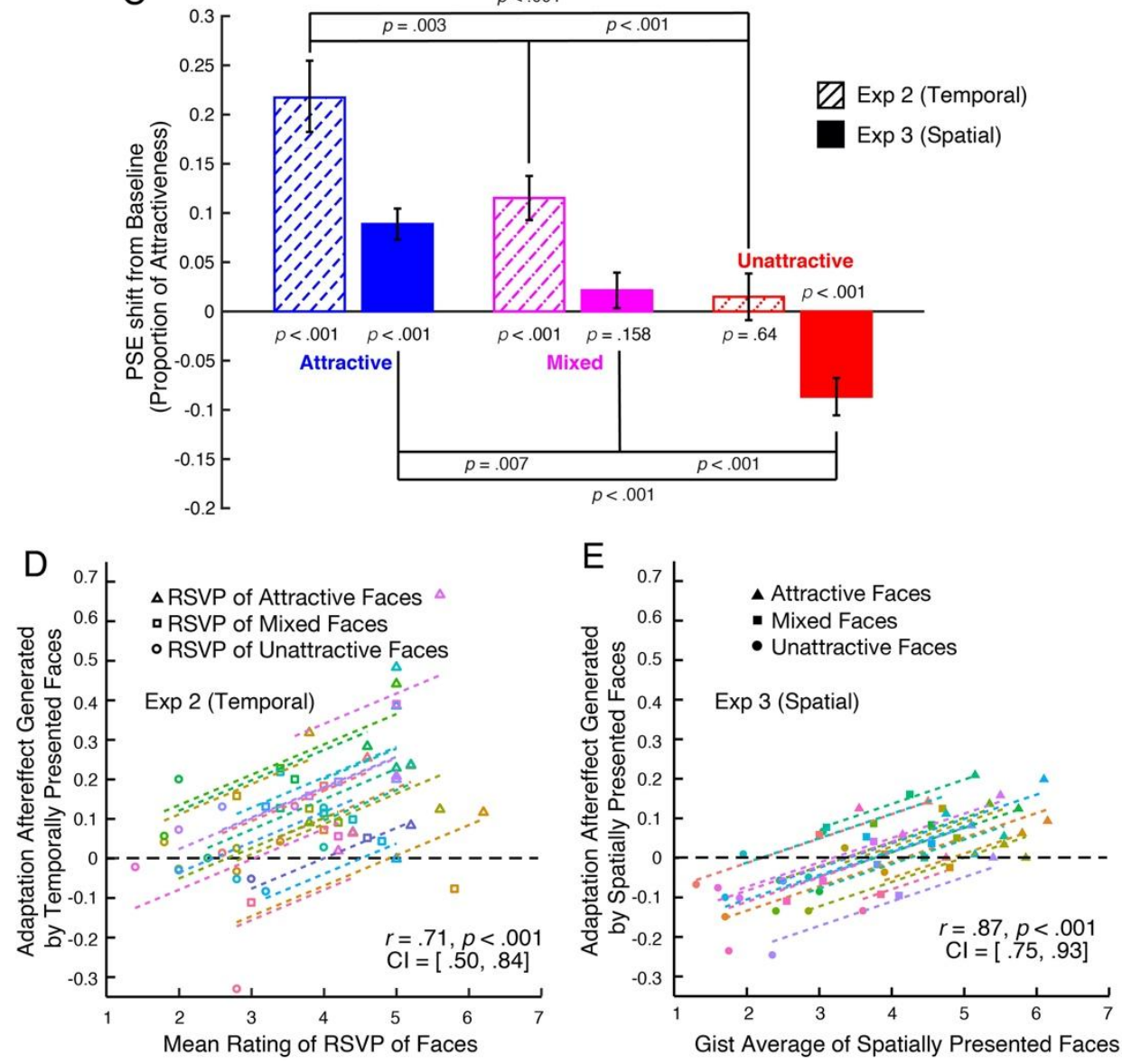

Figure 3. Adaptation aftereffects to temporally presented RSVPs (Experiment 2) and spatially presented faces (Experiment 3). (A) The psychometric functions of Experiment 2's participants averaged together. 'Error bars indicate the standard error of the mean. (B) The psychometric functions of Experiment 3's participants averaged together. (C) Combined summary of all participants' results from Experiment 2 and Experiment 3. The hatched bars indicate Temporal Presentation RSVP conditions (Experiment 2), and the solid bars represent Spatial Presentation conditions (Experiment 3). (D) The adaptation aftereffect as a function of the attractiveness rating of the RSVP of faces in Experiment 2. (E) The adaptation aftereffect as a function of the mean attractiveness rating of the adapting 
faces in Experiment 3. In both (D) \& (E), each color represents the data from one individual participant. The horizontal dashed black auxiliary line indicates no adaptation aftereffect.

In Experiment 2, we replicated the results from Experiment 1, but further illustrated the linear fashion in which the brain morph averages the attractiveness of a temporal stream of attractive, unattractive and mixed faces. These results therefore lend further support to our morph averaging hypothesis for temporally presented face groups. Interestingly, although the ensemble representation of the unattractive face RSVP stream was not processed as unattractive, as reflected by the lack of aftereffects, the direct ratings of these unattractive RSVPs did appear to be perceived as unattractive to some extent $(M=2.65$ out of a 1-to-7 scale, see the above Results section for more details). Previous work has shown that adaptation aftereffects can yield insights into perceptual operations even in the absence of differences in direct ratings (Liu et al., 2014). Thus, adaptation and direct rating may reflect two distinct visual processes: perceptual vs. cognitive process.

\section{Experiment 3: Spatial ensemble statistics represent the gist}

Across Experiments 1 and 2 we have shown temporal ensemble perception extracts the morph average. However, is this also true for spatial ensemble coding when a group of faces is presented simultaneously? We previously showed that the adaptation aftereffects produced by spatially presented faces (i.e., a group presented onscreen at the same time) generated aftereffects in the direction that we would expect if the gist averaging hypothesis was true (Ying et al., 2019); i.e., the unattractive faces made subsequently presented faces appear more attractive, and adapting to a mix of unattractive and attractive faces produced no aftereffects relative to the 
392 baseline no adaptation condition. This result is at odds with the morph averaging that we have

393 observed from our RSVP paradigms in Experiments 1 and 2. We therefore wanted to replicate

394 this gist averaging in a spatial adaptation paradigm by using the same adapting faces from

395 Experiment 2. By using identical adapting faces, we could directly compare the aftereffects

396 derived from temporal and spatial ensemble coding. If the aftereffects between Experiment 2 and

3973 are indistinguishable, then it would imply that a similar mechanism is at work both temporally

398 and spatially; i.e., the faces are being morph averaged from their fiducial points and surface

399 characteristics. However, if the aftereffects between the two experiments are different, then it

400 would provide the first evidence that temporal and spatial ensemble statistics may reflect

401 qualitatively distinct calculations. For example, if gist averaging occurs during spatial ensemble

402 coding, then we would expect an overall negative shift for all of the adapting face conditions

403 relative to those effects observed in Experiment 2: e.g., the unattractive group will now elicit

404 negative aftereffects, the mixed group will be no different from baseline, and the attractive group

405 will elicit smaller positive aftereffects than the attractive group in Experiment 2. We test these

406 hypotheses in Experiment 3.

407

\section{Experiment 3: Methods}

Eighteen new participants (11 Females; Mean Age: 22.78) participated in this

410 experiment; we had initially aimed for 20, but two dropped out during the experiment. Here we

411 used the same adapting faces and blocks from Experiment 2, except the mixed condition only

412 contained two attractive and two unattractive faces so that there were only four faces in the

413 adapting group. During adaptation, the four adapting faces were presented around the central 
414 fixation cross (Figure 4), with the test face presented at the center of the screen. The center-

415 center difference between each adaptor and the central fixation cross is around $3^{\circ}$ (124.5 pixels).

416 This spatial layout is similar to our recent study on ensemble coding of facial attractiveness

417 (Ying et al., 2019). The trial sequence was otherwise similar to Experiments 1 and 2. After the 418 experiment we asked the participants to rate the attractiveness of the eight individual adapting 419 faces to compute an average from the ratings, thereby reflecting the gist average.

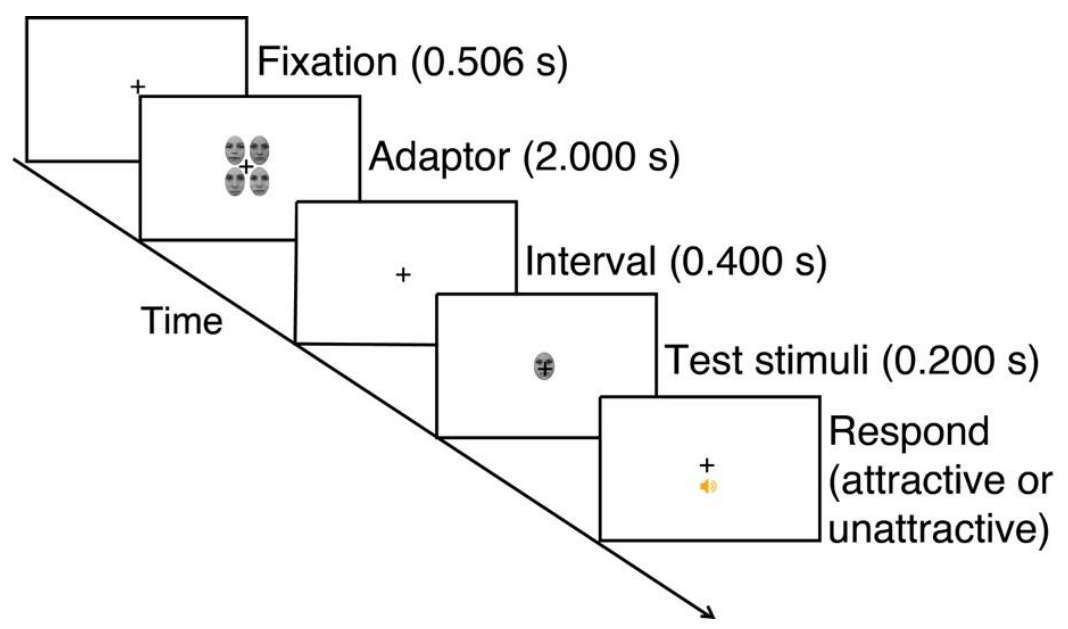

Figure 4. Example trial sequence from a spatial adaptation condition (the demonstrated faces are AF01NES, AF05NES, AF06NES, AF07NES and AF34NES from KDEF database). Participants fixated on the cross at all times. After $0.506 \mathrm{~s}$, four adapting faces simultaneously appeared for $2 \mathrm{~s}$. After a $0.4 \mathrm{~s}$ interval, the test face appeared on the screen for $0.2 \mathrm{~s}$. Then a beep sound indicated participants should judge the attractiveness of the target face by pressing the ' $\mathrm{A}$ ' button for attractive, or the ' $\mathrm{S}$ ' button for unattractive. Experimental parameters for all conditions and experiments are detailed in the Methods section.

\section{Experiment 3: Results and Discussion}

The mean adaptation results from all participants are shown in the psychometric curves in Figure 3B. Unlike Experiments 1 and 2, the Unattractive condition generated a leftward shift away from baseline; this direction is what we would expect if our participants were adapting to the unattractive group as though they were unattractive (Ying et al., 2019). Such differences 
433 relative to Experiment 2 were also observed for the Mixed condition, which failed to generate

434 any significant aftereffects. We statistically examined what aftereffects our spatial conditions

435 produced relative to the baseline condition. Significant aftereffects were generated by both the

436 attractive (Figure 3C, $M=.093, S E M=.016 ; t(17)=5.91, p<.001)$ and unattractive $(M=-.083$,

$437 S E M=.020 ; t(17)=-4.20, p=.001)$ groups. Test faces were rated as unattractive following

438 adaptation to the attractive group, and conversely rated as attractive more frequently following

439 the unattractive groups adaptation, all relative to baseline. By contrast, the mixed faces evoked

440 no aftereffects $(M=.028, S E M=.019 ; t(17)=1.48, p=.16)$.

441 An ANOVA on the three adaptation conditions was significant $(F(2,34)=50.42, p<.001$,

$\left.442 \eta_{\mathrm{p}}{ }^{2}=.75\right)$. Bonferroni corrected comparisons showed that the attractive and unattractive $(t(17)=$

$4438.69, p<.001)$, attractive and mixed $(t(17)=3.56, p=.007)$, and mixed and unattractive $(t(17)=$

$4447.93, p<.001)$ conditions were all significantly different from one another. As in the case of

445 Experiment 2, there was a significant positive repeated measures correlation $(r=.87, p<.001$,

$44695 \%$ CI $[0.75,0.93]$; Figure $3 \mathrm{E})$ between the mean attractiveness ratings of the groups of

447 adapting faces and their aftereffects.

448 A side by side comparison between Experiment 2 and 3 (Figure 3C), shows qualitative

449 differences between the aftereffects of our RSVP experiments and the spatial aftereffects here;

450 note that these differences are apparent despite us using the same adapting faces between the

451 experiments. To confirm these differences statistically, a mixed model ANOVA on the adaptation

452 aftereffects was performed, with a between subject factor of Group (Experiment 2: Temporal vs.

453 Experiment 3: Spatial) and a within subject factor of Attractiveness (unattractive vs. mixed vs.

454 attractive). We found a significant main effect of Attractiveness (with Greenhouse-Geisser

455 correction, $\left.F(1.60,57.46)=73.30, p<.001, \eta_{\mathrm{p}}{ }^{2}=.67\right)$ due to differences between the adaptation 
456 aftereffects (i.e., attractive $>$ mixed $>$ unattractive, Figure $3 \mathrm{~A}$, all $p s<.001$ ). Similarly, there was

457 also a significant main effect of $\operatorname{Group}\left(F(1,36)=12.19, p=.001, \eta_{\mathrm{p}}{ }^{2}=.25\right)$ due to the

458 Experiment 2 Temporal group exhibiting more positive aftereffects in contrast to our current

459 Spatial group $(\operatorname{Exp} 2 M=.12$ vs. $\operatorname{Exp} 3 M=.012)$. Finally, the Group $\times$ Attractiveness was not 460 significant (with Greenhouse-Geisser correction, $F(1.59,57.36)=.80, p=.45, \eta_{\mathrm{p}}{ }^{2}=.02$ ). These

461 findings therefore indicate that while our participants were producing aftereffects that were

462 comparably distinct between attractiveness conditions, the actual perceptual outcomes as

463 reflected by adaptation aftereffects, appeared qualitatively different between Experiments 2 and 4643.

To test whether the above differences in adaptation were also present in the direct ratings,

466 we performed a mixed model ANOVA on the mean attractiveness ratings of the adapting faces

467 with a between subjects factor of Group (Temporal Experiment 2 vs. Spatial Experiment 3) and a

468 within subjects factor of Attractiveness (unattractive vs. mixed vs. attractive). There was a

469 significant main effect of Attractiveness $\left(F(2,72)=302.74, p<.001, \eta_{\mathrm{p}}{ }^{2}=.89\right)$ due to the faces

470 being rated significantly different from one another (i.e., attractive $>$ mixed $>$ unattractive, all $p s$

$471<.001)$, but no main effect of $\operatorname{Group}\left(F(1,36)=.025, p=.88, \eta_{\mathrm{p}}{ }^{2}=.001 ;\right.$ Bayesian analyses

472 provided further support for the null hypothesis; $B F_{01}=4.08$ ). There was, however, a significant

473 interaction between the effects of Attractiveness and $\operatorname{Group}\left(F(2,72)=3.64, p=.031, \eta_{\mathrm{p}}{ }^{2}\right.$

$474=.092$ ). Despite this interaction, there were no significant between group differences in the mean

475 attractiveness ratings of the adapting faces for each of the attractiveness blocks (attractive $p=.11$,

476 mixed $p=.82$, unattractive $p=.43$ ). Thus, presenting the adapting faces spatially or temporally

477 (RSVP) did not change participants' ratings of the adapting faces' attractiveness. These results

478 suggest that the qualitative differences in adaptation aftereffects derived from temporal and 
479 spatial ensemble coding are not due to differences in the perceptions of the adapting faces' 480 attractiveness.

While there were some minor differences between the adaptation durations in

482 Experiments $2 \& 3$, we do not believe that these differences affect our interpretations of the data.

483 Research into the time course of face adaptation has revealed (e.g., facial identity: Rhodes,

484 Jeffery, Clifford, \& Leopold, 2007; facial expression: Burton, Jeffery, Bonner, \& Rhodes, 2016)

485 that adaptation aftereffects follow the classic time course pattern of 'logarithmic build-up' and

486 'exponential decay'. This means that the adaptation aftereffect can be altered quantitatively by

487 some changes in time (like the adaptation duration), but not qualitatively. We recently found that

488 facial expression adaptation aftereffect can be generated after as brief as 34 ms of adaptation

489 (Sou \& Xu, 2019). Thus, the qualitative differences in aftereffects from temporal and spatial

490 ensemble coding here are likely to be maintained, even if the adaptation duration was matched

491 across conditions. To confirm this fact though, we ran a new experiment.

\section{Experiment 4: Spatial-Temporal ensemble statistics induce morph}

494 averaging

While the attractive and unattractive temporal face streams generated asymmetrical

496 aftereffects in Experiment 2 (i.e., the attractive group generated aftereffects, but the unattractive

497 faces did not), the spatial face groups generated symmetrical aftereffects in Experiment 3 (Figure

498 3C, attractive group generated aftereffects, as too did the unattractive group). While there are

499 other minor differences between the procedures across Experiments 2 and 3, such as the

500 locations of the RSVP versus the static spatial adaptor locations, we do not believe these are 
501 causing the qualitative differences we observe between temporal and spatial ensemble coding.

502 Instead, we believe that these effects reflect the fact that temporal and spatial ensemble coding

503 computations are distinctly different. However, to be certain of this belief, we decided to run

504 Experiment 3 again, except this time, we added an RSVP manipulation to the adapting faces.

505 This meant that we could directly compare 'pure' spatial ensemble coding (i.e., that derived from

506 the static groups of faces in Experiment 3) versus temporal ensemble coding (i.e., that derived

507 from the RSVP of faces presented at the same four locations as the static spatial groups).

508 Furthermore, we had participants directly rate the mean attractiveness of the groups of

509 adapting faces in both the spatial and temporal conditions so that we could assess whether the

510 direct rating and the adaptation measures of ensemble coding were similar across presentation

511 methods.

512

\section{Experiment 4: Methods}

Twenty new participants (13 Females; Mean Age: 21.75) participated in this experiment.

515 We matched the sample size of the current experiment with the previous two experiments. The

516 general design was adapted from Experiments 2 and 3. The trial sequence was similar to that of

517 Experiment 3. During adaptation, there were four RSVP face streams simultaneously presented

518 surrounding the central fixation cross (Figure 5). The spatial locations of the four streams were

519 identical to those in Experiment 3 ( $3^{\circ}$ away from the fixation cross). Thus, we name this

520 manipulation the Spatial-Temporal condition. Within each RSVP stream, the faces were

521 presented at $42.5 \mathrm{~Hz}$ (the same as Experiments 1 and 2) for $1.98 \mathrm{~s}$ (84 faces in total, and each

522 presented for $23.5 \mathrm{~ms}$; the adaptation duration is almost identical to Experiment 3: $2 \mathrm{~s}$ ). All of the 
523 faces presented within the Spatial-Temporal streams were the faces used in Experiment 3, with

524 'ATT', 'MIX', and 'UNA' conditions. These faces were presented in a pseudo-random order, so

525 that within each frame, the four faces presented onscreen together were always of different

526 identities.

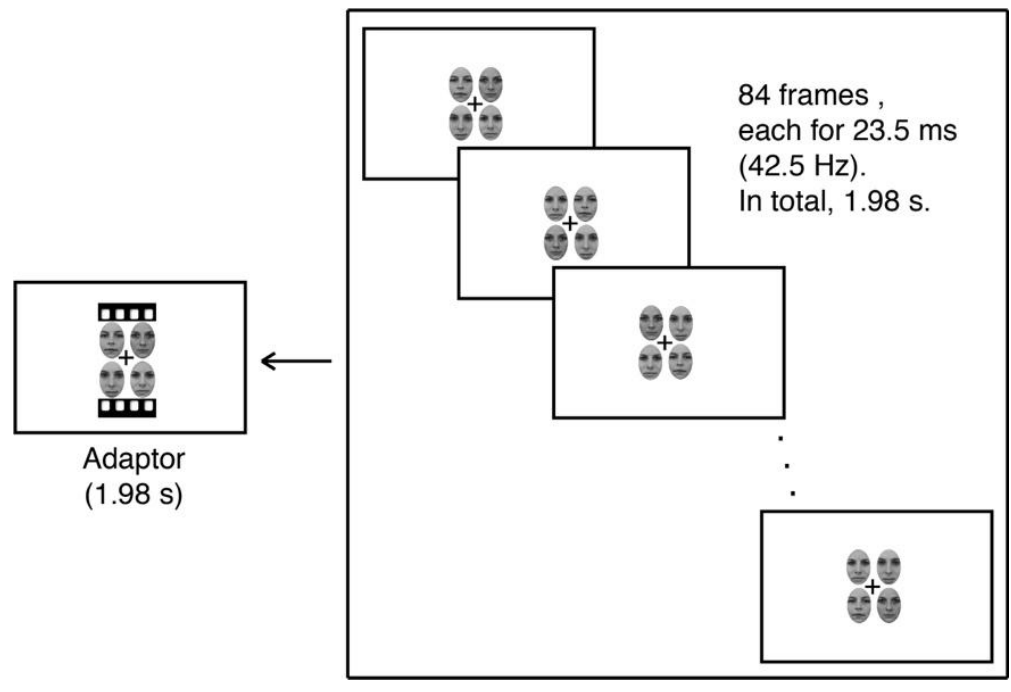

Figure 5. The Spatial-Temporal adaptor for Experiment 4 (the demonstrated faces are AF01NES, AF05NES, AF06NES, AF07NES and AF34NES from KDEF database). The adaptor is four simultaneous streams of RSVPs of faces $(42.5 \mathrm{~Hz}$, the same as Experiment 2), presented for $1.98 \mathrm{~s}$ in total. The spatial relationships of the four streams ( $3^{\circ}$ away from the central fixation cross) were the same as that in Experiment 3. Thus, the Spatial-

532 Temporal adaptor is a combination of the adaptation manipulations from Experiments 2 and 3. attractiveness via direct ratings. We asked our participants to rate the attractiveness of each

536 adapting face, and these adapting faces as a group in the spatial-temporal configuration on a 7-

537 point scale. Each group of faces was presented for $1 \mathrm{~s}$. We chose $1 \mathrm{~s}$ for direct rating because it

538 has been shown that this is sufficiently long for the participants to make judgments on

539 attractiveness (e.g., Ying et al., 2019). Moreover, to clarify whether the computer-generated

540 averaged face is indeed more attractive than the mean of its components, we also asked 
541 participants to rate the computer-generated averaged face of the attractive and unattractive

542 groups. The order of the stimuli in direct rating tasks was randomized for each participant.

\section{Experiment 4: Results and Discussion}

The mean adaptation results from all participants are summarized in Figure 6A. After

exposure to the attractive Spatial-Temporal faces (blue dotted line), there was a rightward shift in

547 the psychometric curve relative to baseline, indicating that the ensemble representation of this

548 group is attractive. A similar shift, albeit smaller in magnitude, is observed in the 'MIX'

549 condition (magenta dash-dotted line). By contrast, the 'UNA' condition (red dotted line) failed to

550 generate a significant shift from the baseline condition. This finding replicates our temporal

551 ensemble coding results in Experiment 2, and appears qualitatively different from the aftereffects

552 induced via spatial adaptation in Experiment 3.

Overall, significant aftereffects were generated by the Spatial-Temporal attractive (Figure

$5546 \mathrm{~B}, M=.16, S E M=.029 ; t(19)=5.57, p<.001)$ and mixed $(M=.068, S E M=.022 ; t(19)=2.97$,

$555 p=.008)$ but not the unattractive $(M=-.013, S E M=.02 ; t(19)=-.85, p=.41)$ faces. Bayesian

556 analyses suggested that the lack of aftereffects in the unattractive Spatial-Temporal condition

557 was in favor of the null hypothesis $\left(B F_{01}=3.122\right)$. Thus, the observed data indicates that there

558 was indeed no adaptation aftereffect in the unattractive Spatial-Temporal condition. To compare

559 the three adaptation conditions, we conducted an ANOVA and found significant differences

560 among all three adaptation conditions (with Greenhouse-Geisser correction, $F(1.47,27.98)=$

$\left.56126.36, p<.001, \eta_{\mathrm{p}}{ }^{2}=.58\right)$. Subsidiary Bonferroni corrected comparisons showed significant

562 differences between the attractive and unattractive $(t(19)=5.85, p<.001)$, attractive and mixed 
$563(t(19)=3.92, p=.003)$, and mixed and unattractive $(t(19)=4.82, p<.001)$ conditions. These

564 findings confirm our hypothesis that temporal ensemble coding induces morph averaging,

565 whereas ensemble coding for spatially presented face groups (i.e., Experiment 3) results in gist

566 averaging.

A

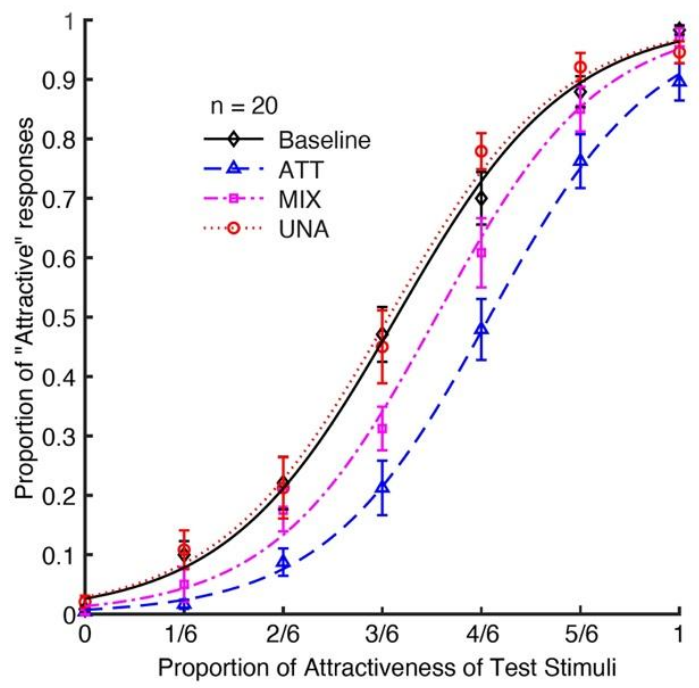

C

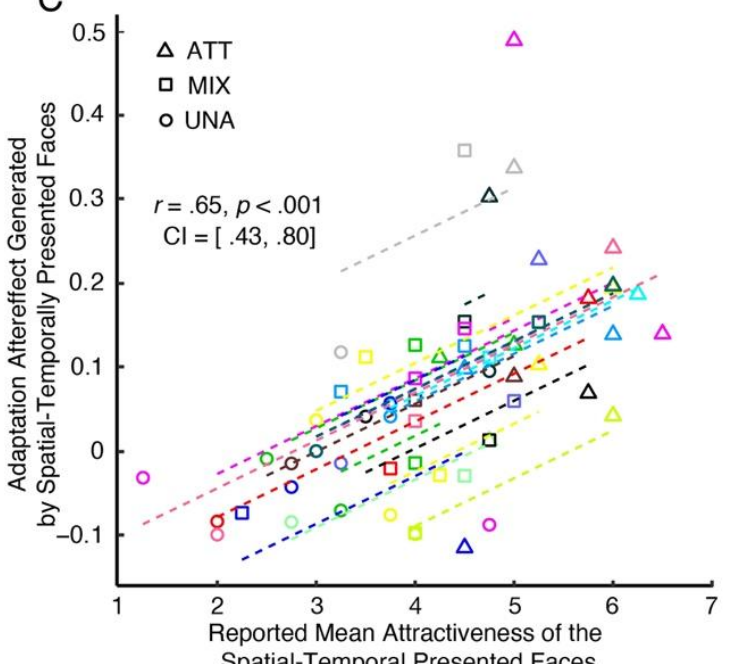

B

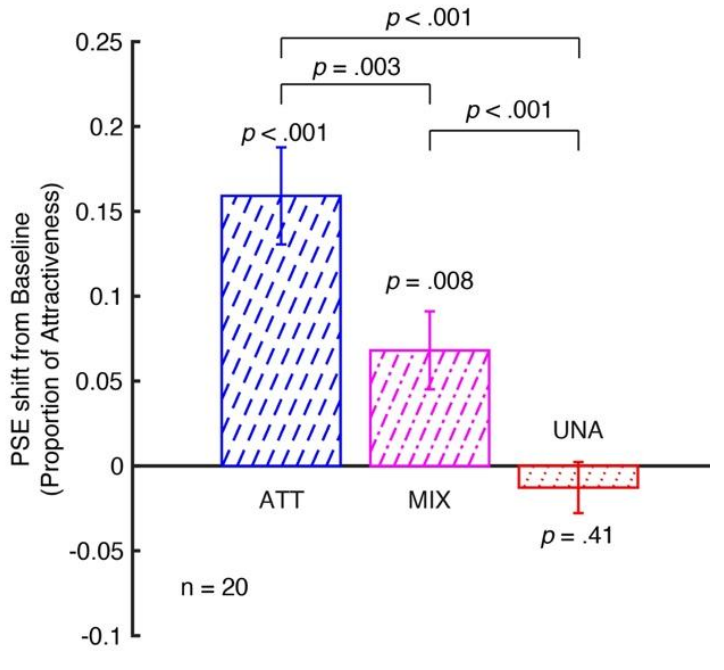

Figure 6. Spatial-Temporal adaptation aftereffects (Experiment 4). (A) The psychometric functions of all participants averaged together. Error bar indicates the SEM. (B) Summary of all 20 participants' results from Experiment 4. (C) The adaptation aftereffect as a function of the reported mean attractiveness of the adapting faces 
To directly test whether the RSVP spatial manipulation we employed here was similar to

574 the effects observed from the RSVP streams in Experiment 2, we ran an ANOVA on the

575 aftereffects of Experiments 2 and 4, with Group (Exp 2, Exp 4) being the between subject factor,

576 and Attractiveness (ATT, MIX, UNA) being the within subject factor. The results showed that

577 there were no significant differences between these two experiments $(F(1,38)=2.20, p=.15$,

$578 \eta_{\mathrm{p}}^{2}=.055$ ), nor any interaction between them and the attractiveness of the faces (with

579 Greenhouse-Geisser correction, $\left.F(1.51,57.47)=.65, p=.49, \eta_{\mathrm{p}}{ }^{2}=.017\right)$. Instead, there was only

580 a significant difference among the three attractiveness conditions (with Greenhouse-Geisser

581 correction, $\left.F(1.51,57.47)=59.51, p<.001, \eta_{\mathrm{p}}{ }^{2}=.61\right)$. Thus, the aftereffects induced by a single

582 RSVP stream (Experiment 2) and multiple RSVP streams (Experiment 4) were comparable, and

583 reflective of morph averaging.

To confirm that temporal and spatial ensemble coding reflect distinct perceptual

585 outcomes, we compared the aftereffects between Experiments 3 and 4 using the same ANOVA.

586 While we did not find any significant interaction between the experiments and the attractiveness

587 of the faces (with Greenhouse-Geisser correction, $F(1.56,55.97)=.58, p=.52, \eta_{\mathrm{p}}{ }^{2}=.016$ ), there

588 was a significant difference among three attractiveness conditions (with Greenhouse-Geisser

589 correction, $\left.F(1.56,55.97)=66.85, p<.001, \eta_{\mathrm{p}}{ }^{2}=.65\right)$. However, in addition, there was also a

590 significant difference between the two experiments $\left(F(1,36)=6.07, p=.019, \eta_{\mathrm{p}}{ }^{2}=.14\right)$; the

591 Spatial-Temporal aftereffects in Experiment 4 were more positive than those induced by the

592 spatial group in Experiment 3. Taken together, the pattern of observed aftereffects in Spatial-

593 Temporal adaptation is more similar to temporal ensemble coding, than to the static spatial

594 ensemble coding we observed in Experiment 3. In other words, the Spatial-Temporal ensemble is

595 largely driven by morph averaging of the faces from the temporal streams. 
To examine the attractiveness ratings of the adapting faces directly, we conducted an

597 ANOVA on the participants' attractiveness ratings of the Spatial-Temporal streams and found a

598 significant difference among the three types of attractiveness adaptors $(F(2,38)=47.72, p<.001$,

$\left.599 \eta_{\mathrm{p}}^{2}=.72\right)$. Further comparisons revealed that participants rated the Spatial-Temporal streams of

600 the attractive faces $(M=5.38, S E M=.015)$ as the most attractive, followed by the Spatial-

601 Temporal streams of mixed faces $(M=4.16, S E M=.015)$, with the Spatial-Temporal streams of

602 unattractive faces $(M=3.20, S E M=.020)$ being rated as least attractive (all $p s<.001)$. We

603 further compared the direct ratings between Experiments 3 and 4 with a mixed-model ANOVA.

604 There was a significant difference among the three attractiveness conditions, as expected (with

605 Greenhouse-Geisser correction, $\left.F(1.43,51.30)=169.33, p<.001, \eta_{\mathrm{p}}{ }^{2}=.83\right)$. Importantly, there

606 was also a significant difference between the two experiments $\left(F(1,36)=4.42, p=.043, \eta_{\mathrm{p}}{ }^{2}\right.$

$607=.11)$; the spatial-temporal streams $(\operatorname{Exp} 4)$ were rated as more attractive than the 'spatial group'

608 (Exp 3). Thus, both rating and adaptation aftereffects data suggest that spatial (Exp 3) and

609 spatial-temporal (Exp 4) ensemble coding are distinct from each other. There was no significant

610 interaction between the experiments and the attractiveness of the faces (with Greenhouse-Geisser

611 correction, $\left.F(1.43,51.30)=2.74, p=.09, \eta_{\mathrm{p}}^{2}=.071\right)$.

612 Why were there similar ratings between Experiments 2 and 3, but different ratings

613 between Experiments 3 and 4? We believe the reason was in the tasks in rating. In Experiment 2,

614 'mean attractiveness' was measured by participants rating the mean attractiveness of each RSVP

615 stream; while in Experiment 3, the 'gist/mean attractive' was measured by the mean rating of

616 individual adapting faces by another group of participants. By contrast, in Experiment 4, 'mean

617 attractiveness' was measured by participants rating the mean attractiveness of Spatial-Temporal 
619 task should be conducted.

621 significant positive correlation between the attractiveness ratings of the Spatial-Temporal streams 622 and the adaptation aftereffects (Figure $6 \mathrm{C}, r=.65, p<.001,95 \% \mathrm{CI}[0.42,0.80]$ ). This indicates

623 that the observed attractiveness aftereffects were driven by the ensemble coding of the

624 attractiveness of the adapting stimuli. components, we compared the mean ratings of individual attractive $(M=4.35, S E M=.18)$ and unattractive $(M=1.92, S E M=.19)$ faces with their computer-generated average faces (attractive: $M=5.65, S E M=.19$; unattractive: $M=2.59, S E M=.17$ ) conditions. We found that in both the attractive $(t(19)=7.46, p<.001)$ and unattractive $(t(19)=5.58, p<.001)$ conditions, the computer-generated average faces were more attractive than their components.

\section{General Discussion}

634 four experiments. Experiment 1 showed that RSVP streams and their paired computer-generated

635 morphed averages led to comparable, and correlated, facial attractiveness aftereffects.

636 Experiment 2 replicated the findings from Experiment 1, thereby further supporting the morph 637 average hypothesis; i.e., no aftereffects in the unattractive condition, such that the unattractive 638 group was perceived as more attractive than the gist of the group (i.e., these faces are 639 unattractive), and positive aftereffects in the mixed condition. Moreover, in Experiment 2 we 
640 found that aftereffects increased as a function of the underlying RSVP stream's attractiveness,

641 suggesting that temporal ensemble perception occurs in a linear fashion. In contrast to the first

642 two experiments, however, Experiment 3 showed that spatial ensemble statistics favored the gist

643 averaging hypothesis; i.e., no aftereffects in the mixed condition, and negative aftereffects in the

644 unattractive condition. Combining the manipulations in Experiments 2 (temporal) and 3 (spatial)

645 together, Experiment 4 showed that ensemble coding of a Spatial-Temporal presentation of faces

646 is formed by morph averaging, and not the gist. This confirms that the observed differences

647 between Experiments 2 and 3 were not driven by the minor differences in presentation formats,

648 but by distinct ensemble coding operations. Taking all four experiments together, it is clear that

649 temporal and spatial ensemble statistics stem from qualitatively different extraction processes.

650 While a number of prior studies have examined spatial ensemble coding and temporal

651 ensemble coding (Haberman et al., 2015; Haberman \& Whitney, 2007, 2009; Whitney \& Levi,

652 2011; Whitney \& Yamanashi Leib, 2017; Wolfe et al., 2015; Ying \& Xu, 2017; Ying et al., 2019),

653 no study to our knowledge has compared the effects of both. Moreover, even if researchers had

654 compared the averaging of facial traits other than attractiveness (e.g., emotion) across these two

655 presentation formats, it would have been highly unlikely that they would have observed

656 differences between temporal and spatial ensemble coding anyway. This is because adapting to

657 facial emotion, via either a morph or gist averaging process, would result in the same outcome

658 (as illustrated in Figure 7A with hypothetical data). Here, we took advantage of the fact that

659 averaging faces together from their morphed properties makes them more attractive (DeBruine et

660 al., 2007; Leder, Goller, Forster, Schlageter, \& Paul, 2017; Perrett et al., 1994; Valentine et al.,

661 2004; as illustrated in Figure 7B with hypothetical data). By doing so, we confirmed that there 
662 are qualitative differences between how ensemble coding mechanisms extract distinct

663 information across spatial and temporal presentations.

A

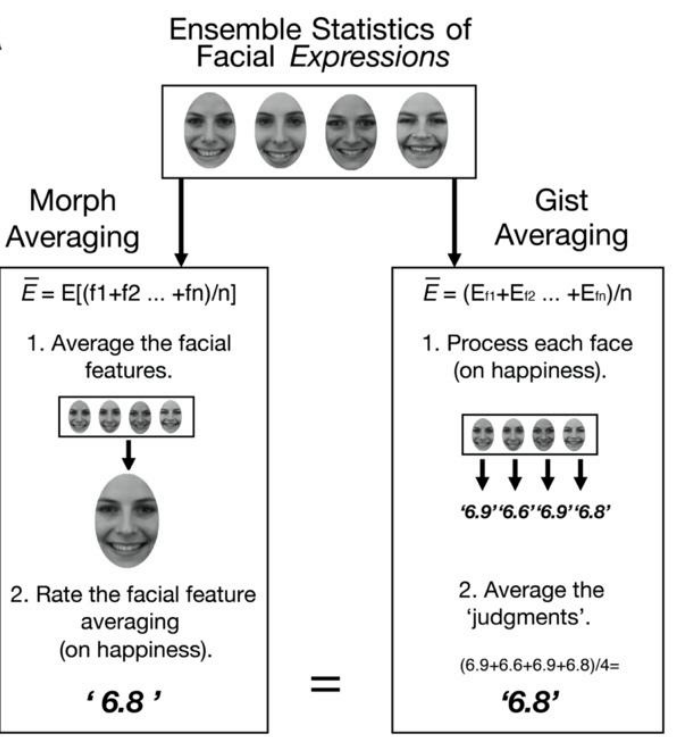
3.8 versus 2 ).

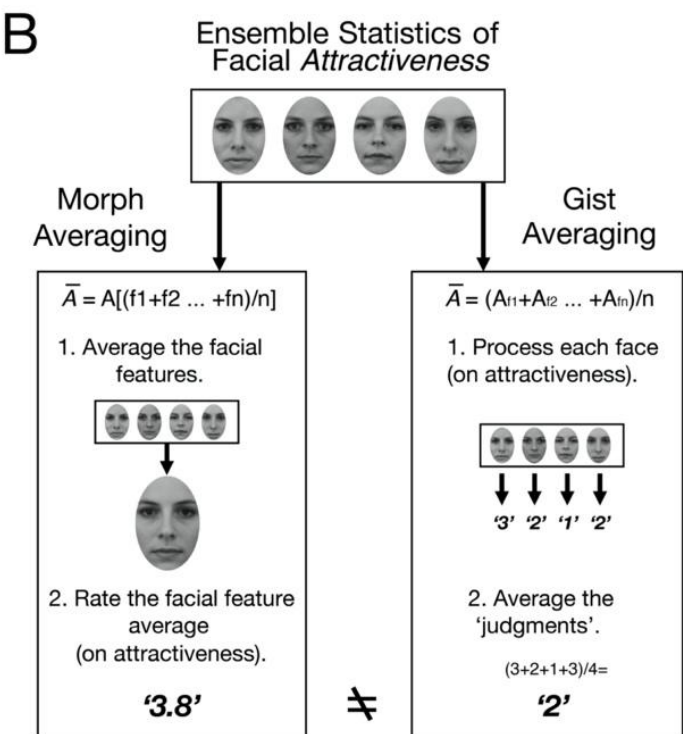

Figure 7. The 'morph averaging' and 'gist averaging' hypotheses (the demonstrated faces are AF01NES, AF05NES, AF06NES and AF07NES from KDEF database; the digits are from hypothetical data only for demonstration purposes). (A) Ensemble coding for facial expressions: the 'morph averaging' and 'gist averaging' hypotheses predict the same perceptual outcome for emotion; i.e., happy intensity rating of 6.8. (B) Ensemble coding of facial attractiveness: the 'morph averaged' face is more attractive than the mean attractiveness of its individual component faces, with the averaged face not equal to the mean 'judgments' (i.e., attractiveness rating of

We should explicitly clarify to readers that the null results found in Experiments 1, 2 and 4 (i.e., in the unattractiveness conditions) actually support our morph averaging hypothesis of temporal ensemble coding. These findings were not due to the unattractive faces not being unattractive enough to elicit negative aftereffects, nor are the lack of effects due to a lack of power. First, we used the very same stimuli in Experiments 2, 3, and 4, with the presentation methods being the largely the only difference among the 3 studies. The negative aftereffects generated by the unattractive condition in Experiment 3 shows that the unattractive group was

680 processed by the participants as unattractive; i.e., the participants perceived the subsequently 
681 presented faces as attractive (which replicates the results from Ying et al., 2019). In other words,

682 the very same faces generated asymmetrical aftereffects when being presented temporally, but 683 generated symmetrical aftereffects when presented spatially.

684 This finding is at odds with the suggestion that the unattractive adapting faces in 685 Experiments 1,2, and 4 were simply insufficient in unattractiveness to elicit the aftereffects 686 expected from an unattractive group. This point is further strengthened by the large effect size in 687 the unattractive condition's aftereffects in Experiment 3. Moreover, by analyzing the data via 688 Bayes Factors (Dienes, 2014; Rouder et al., 2009), we found evidence supporting the null 689 hypothesis (i.e., the unattractive RSVP faces are equivalent to baseline and their computer690 generated average face), thus countering any suggestion that the null effects across Experiments 6911,2 , and 4 were a result of low statistical power. Simply put, the current data strongly favors the 692 notion that the RSVP streams of unattractive faces are perceived as neither attractive nor 693 unattractive relative to participants' baseline norms of attractiveness, and that this perceptual 694 outcome was not due to these faces not being unattractive enough to elicit negative aftereffects. 695 Instead, participants must have been averaging the unattractive RSVP stream in such a fashion 696 that it made the faces be processed as more attractive than their underlying gist (i.e., unattractive). 697 This was clarified by the fact that the aftereffects of the RSVP streams were equivalent to, and 698 correlated with, their computer-generated averaged morph face counterparts. The qualitative differences between the adaptation aftereffects produced by RSVP

700 streams and spatial presentations of faces likely reveal the hierarchical nature of the human face 701 perception system (Bartolomeo, Vuilleumier, \& Behrmann, 2015; Behrmann \& Plaut, 2013;

702 Duchaine \& Yovel, 2015; Eimer, 2000; Gobbini \& Haxby, 2007; Haxby, Hoffman, \& Gobbini, 703 2000, 2002; Haxby \& Gobbini, 2012; Liu, Harris, \& Kanwisher, 2002; Young \& Bruce, 2011; 
704 Zhao, Zhen, Liu, Song, \& Liu, 2017). For example, extracting the morph averaging properties of 705 a face arguably occurs at an earlier stage of encoding (Eimer, 2000; Gauthier et al., 2000; Grill-

706 Spector, Knouf, \& Kanwisher, 2004; Kanwisher, McDermott, \& Chun, 1997; Kanwisher \&

707 Yovel, 2006; Pitcher, Walsh, Yovel, \& Duchaine, 2007) in comparison to when the brain can

708 conceptually calculate the aspects of a face that make it unattractive (i.e., gist; O'Doherty et al.,

709 2003). If we consider the visual features processing as perceptual, and the assessment of

710 attractiveness as cognitive, we therefore provide the first direct evidence for distinct ensemble

711 processing of temporal and spatial stimuli such that ensemble coding for temporal stimuli occurs

712 at a perceptual level, whereas ensemble coding for spatial stimuli occurs at a cognitive level.

713 This spatial process may be based on 'local support' such that "data coming from spatially local

714 components of the image tend to use parallel computations, rather than global or serial methods"

715 (e.g., Firestone \& Scholl, 2016; Pylyshyn, 1999; Dawson \& Pylyshyn, 1988; Marr \& Poggio,

716 1979; Rosenfeld, Hummel, \& Zucker, 1976). On the other hand, the refresh rate of the RSVP and

717 spatial-temporal conditions in our experiments are really high. However, we are yet sure that

718 whether the temporal (morph) averaging occurs before or after the attractiveness of the

719 individual faces has been determined. We suspect that a new face norm is continuously being

720 updated as each face is presented in the RSVP stream, and its information extracted. Only once

721 this information has been extracted in the form of a new morphed face norm, can it then produce

722 a conceptual appraisal (e.g., this group of unattractive faces' information morphs together to then

723 be judged as moderately attractive) that drives subsequent adaptation aftereffects. We anticipate

724 future neuroimaging and electrophysiological work will confirm these distinct neural stages

725 responsible for driving ensemble statistics derived from temporal versus spatial averaging. 


\section{Conclusions}

Researchers have long speculated as to the composition of the neural calculations

729 performed during ensemble coding. We have shown for the first time that temporal ensemble

730 statistics do not simply reflect the 'gist' of the attractiveness judgements attributed to a group of

731 faces, but are instead extracted by morph averaging the group's fiducial points and surface

732 characteristics together. By contrast, spatial ensemble coding appears reflective of a gist

733 averaging process in which the group's general characteristics of attractiveness (e.g., this group

734 is unattractive), can be maintained. This reveals two distinct levels of ensemble statistics that can

735 occur for the same facial trait: the gist averaging we observed during static spatial ensemble

736 coding, and the morph averaging for temporal ensemble coding. We anticipate that these results

737 will help inform a broader theoretical framework to understand ensemble perception, but also

738 enhance our knowledge of face processing and appraisal mechanisms.

\section{Author Contributions}

H. Ying, A. Choo, E. Burns and H. Xu developed the study concept and contributed to the

742 study design. H. Ying and A. Choo performed testing and data collection. H. Ying performed the

743 data analysis and interpretation under the supervision of H. Xu. H. Ying drafted the manuscript,

744 and E. Burns and H. Xu provided critical revisions. All authors approved the final version of the

745 manuscript for submission. 


\section{Acknowledgements}

Supported by Nanyang Technological University Research Scholarship (HY),

751 Undergraduate Research Experience on Campus (AC), College of Arts, Humanities and Social

752 Sciences Incentive Scheme (HX), and Singapore Ministry of Education Academic Research

753 Fund (AcRF) Tier 1 (HX). H. Ying is also supported by the Social Science Foundation of Jiangsu

754 Province (17JYC006), the City \& University strategy-Soochow University Leading Research

755 Team in Humanities and Social Sciences. Parts of this research (data from Exp 1) were presented

756 at the Annual Meeting of Visual Science Society (VSS), May 2017, St. Pete Beach, Florida. The

757 research reported here forms part of H. Ying's Ph.D. thesis at Nanyang Technological University.

758 All data have been made publicly available via the Open Science Framework (OSF) and can be

759 accessed at https://osf.io/rgdja/.

\section{References}

1. Alvarez, G. A. (2011). Representing multiple objects as an ensemble enhances visual cognition. Trends Cogn Sci, 15(3), 122-131. doi:10.1016/j.tics.2011.01.003

2. Ariely, D. (2001). Seeing sets: Representation by statistical properties. Psychological science, 12(2), 157-162. doi:Doi 10.1111/1467-9280.00327

3. Bachy, R., \& Zaidi, Q. (2014). Factors governing the speed of color adaptation in foveal versus peripheral vision. JOSA A, 31(4), A220-A225.

4. Bartolomeo, P., Vuilleumier, P., \& Behrmann, M. (2015). The whole is greater than the sum of the parts: Distributed circuits in visual cognition. Cortex, 72, 1-4. doi:10.1016/j.cortex.2015.09.001

5. Bakdash, J. Z., \& Marusich, L. R. (2017). Repeated measures correlation. Frontiers in psychology, 8, 456. doi: 10.3389/fpsyg.2017.00456.

6. Behrmann, M., \& Plaut, D. C. (2013). Distributed circuits, not circumscribed centers, mediate visual recognition (vol 17, pg 210, 2013). Trends in Cognitive Sciences, 17(7), 361-361. doi:10.1016/j.tics.2013.05.009

7. Brainard, D. H. (1997). The Psychophysics Toolbox. Spat Vis, 10(4), 433-436.

8. Burns, E. J., Martin, J., Chan, A. H. D., \& Xu, H. (2017). Impaired processing of facial happiness, with or without awareness, in developmental prosopagnosia. Neuropsychologia, 102, 217-228. doi:10.1016/j.neuropsychologia.2017.06.020 
9. Burton, N., Jeffery, L., Bonner, J., \& Rhodes, G. (2016). The timecourse of expression aftereffects. Journal of Vision, 16(15), 1-1.

10. Chen, C., Chen, X., Gao, M., Yang, Q., \& Yan, H. (2015). Contextual influence on the tilt after-effect in foveal and para-foveal vision. Neuroscience Bulletin, 31(3), 307-316. Computational processes in human vision: An interdisciplinary perspective, ed. Z. W. Pylyshyn. Ablex Publishing.

12. Debruine, L., Jones, B. C., Unger, L., \& Little, A. C. (2007). Dissociating averageness and attractiveness: attractive faces are not always average. Journal of Experimental Psychology: Human Perception and Performance, 33(6), 11. doi:0.1037/00961523.33.6.1420.

13. Debruine, L., \& Tiddeman, B. (2017). WebMorph., Retrieved from http://webmorph.org/.

14. Dienes, Z. (2014). Using Bayes to get the most out of non-significant results. Frontiers in Psychology, 5. doi:ARTN 78110.3389/fpsyg.2014.00781

15. Duchaine, B., \& Yovel, G. (2015). A Revised Neural Framework for Face Processing. Annual Review of Vision Science, Vol 1, 1, 393-416. doi:10.1146/annurev-vision-082114035518

16. Elias, E., Dyer, M., \& Sweeny, T. D. (2017). Ensemble Perception of Dynamic Emotional Groups. Psychological Science, 28, 193-203. doi:10.1177/0956797616678188

17. Eimer, M. (2000). The face-specific N170 component reflects late stages in the structural encoding of faces. Neuroreport, 11(10), 2319-2324.

18. Faul, F., Erdfelder, E., Buchner, A., \& Lang, A.-G. (2009). Statistical power analyses using $\mathrm{G}^{*}$ Power 3.1: Tests for correlation and regression analyses. Behavior Research Methods, 41, 1149-1160.

19. Firestone, C., \& Scholl, B. J. (2016). Cognition does not affect perception: Evaluating the evidence for "top-down" effects. Behavioral and Brain Sciences, 39, e229.

20. Fox, C. J., \& Barton, J. J. (2007). What is adapted in face adaptation? The neural representations of expression in the human visual system. Brain research, 1127, 80-89.

21. Galton, F. (1878). Composite portraits. Journal of the Anthropological Institute of Great Britain \& Ireland, 8, 132-144.

22. Gauthier, I., Tarr, M. J., Moylan, J., Skudlarski, P., Gore, J. C., \& Anderson, A. W. (2000). The fusiform "face area" is part of a network that processes faces at the individual level. Journal of Cognitive Neuroscience, 12(5), 912-912.

23. Gobbini, M. I., \& Haxby, J. V. (2007). Neural systems for recognition of familiar faces. Neuropsychologia, 45(1), 32-41. doi:10.1016/j.neuropsychologia.2006.04.015

24. Grill-Spector, K., Knouf, N., \& Kanwisher, N. (2004). The fusiform face area subserves face perception, not generic within-category identification. Nature Neuroscience, 7(5), 555-562. doi:10.1038/nn1224

25. Haberman, J., Brady, T. F., \& Alvarez, G. A. (2015). Individual differences in ensemble perception reveal multiple, independent levels of ensemble representation. $J$ Exp Psychol Gen, 144(2), 432-446. doi:10.1037/xge0000053

26. Haberman, J., Lee, P., \& Whitney, D. (2015). Mixed emotions: Sensitivity to facial variance in a crowd of faces. Journal of vision, 15(4), 16-16. doi:10.1167/15.4.16

27. Haberman, J., \& Whitney, D. (2007). Rapid extraction of mean emotion and gender from sets of faces. Curr Biol, 17(17), R751-753. doi:10.1016/j.cub.2007.06.039 
825

28. Haberman, J., \& Whitney, D. (2009). Seeing the mean: ensemble coding for sets of faces. J Exp Psychol Hum Percept Perform, 35(3), 718-734. doi:10.1037/a0013899

29. Haberman, J., \& Whitney, D. (2012). Ensemble Perception:summarizing the scene and broadening the limits of visual processing.

30. Haxby, J. V., Hoffman, E. A., \& Gobbini, M. I. (2000). The distributed human neural system for face perception. Trends in Cognitive Sciences, 4(6), 223-233. doi:Doi 10.1016/S1364-6613(00)01482-0

31. Haxby, J. V., Hoffman, E. A., \& Gobbini, M. I. (2002). Human neural systems for face recognition and social communication. Biological Psychiatry, 51(1), 59-67. doi:Doi 10.1016/S0006-3223(01)01330-0

32. Haxby, J. V., \& Gobbini, M. I. Distributed Neural Systems for Face Perception, Oxford University Press (2011).

33. Hsu, S. M., \& Young, A. (2004). Adaptation effects in facial expression recognition. Visual Cognition, 11(7), 871-899.

34. Kanwisher, N., McDermott, J., \& Chun, M. M. (1997). The fusiform face area: A module in human extrastriate cortex specialized for face perception. Journal of Neuroscience, 17(11), 4302-4311.

35. Kanwisher, N., \& Yovel, G. (2006). The fusiform face area: a cortical region specialized for the perception of faces. Philosophical Transactions of the Royal Society B-Biological Sciences, 361(1476), 2109-2128. doi:10.1098/rstb.2006.1934

36. Keysers, C., Xiao, D. K., Foldiak, P., \& Perrett, D. I. (2001). The speed of sight. Jouranl of Cogntive Neuroscience, 13(1), 90-101.

37. Leder, H., Goller, J., Forster, M., Schlageter, L., \& Paul, M. A. (2017). Face inversion increases attractiveness. Acta psychologica, 178, 25-31.

38. Leopold, D. A., O'Toole, A. J., Vetter, T., \& Blanz, V. (2001). Prototype-referenced shape encoding revealed by high-level aftereffects. Nature Neuroscience, 4(1), 89-94. doi:10.1038/82947

39. Liu, J., Harris, A., \& Kanwisher, N. (2002). Stages of processing in face perception: an MEG study. Nat Neurosci, 5(9), 910-916. doi:10.1038/nn909

40. Liu, P., Montaser-kouhsari, L., \& Xu, H. (2014). Effects of face feature and contour crowding in facial expression adaptation. Vision Research, 105, 189-198. https://doi.org/10.1016/j.visres.2014.10.014

41. Luo, C., Burns, E., \& Xu, H. (2017). Association between autistic traits and emotion adaptation to partially occluded faces. Vision research, 133, 21-36.

42. Lundqvist, D., Flykt, A., \& Öhman, A. (1998). The Karolinska directed emotional faces (KDEF). CD ROM from Department of Clinical Neuroscience, Psychology section, Karolinska Institutet, 91-630.

43. Marr, D., \& Poggio, T. (1979). A computational theory of human stereo vision. Proceedings of the Royal Society of London. Series B. Biological Sciences, 204(1156), 301-328.

44. McKeeff, T. J., Remus, D. A., \& Tong, F. (2007). Temporal limitations in object processing across the human ventral visual pathway. Journal of Neurophysiology, 98(1), 382-393. doi:10.1152/jn.00568.2006

45. Morey, R. D., \& Rouder, J. N. (2015). BayesFactor (Version 0.9.11-3)[Computer software]. 
46. O’Doherty, J., Winston, J., Critchley, H., Perrett, D., Burt, D. M., \& Dolan, R. J. (2003). Beauty in a smile: the role of medial orbitofrontal cortex in facial attractiveness. Neuropsychologia, 41(2), 147-155. doi:10.1016/s0028-3932(02)00145-8

47. Pegors, T. K., Mattar, M. G., Bryan, P. B., \& Epstein, R. A. (2015). Simultaneous perceptual and response biases on sequential face attractiveness judgments. Journal of Experimental Psychology: General, 144(3), 664.

48. Pelli, D. G. (1997). The VideoToolbox software for visual psychophysics: Transforming numbers into movies. Spatial vision, 10(4), 437-442.

49. Perrett, D. I., May, K. A., \& Yoshikawa, S. (1994). Facial shape and judgements of female attractiveness. Nature, 368, 239-242. doi:10.1038/368239a0

50. Pitcher, D., Walsh, V., Yovel, G., \& Duchaine, B. (2007). TMS evidence for the involvement of the right occipital face area in early face processing. Current Biology, 17(18), 1568-1573. doi:10.1016/j.cub.2007.07.063

51. Potter, M. C. (1976). Short-term conceptual memory for pictures. J Exp Psychol Hum Learn, 2(5), 509-22. doi: 10.1037/0278-7393.2.5.509

52. Pylyshyn, Z. (1999). Is vision continuous with cognition?: The case for cognitive impenetrability of visual perception. Behavioral and brain sciences, 22(3), 341-365.

53. R Core Team (2017). $R$ : A language and environment for statistical computing. $R$ Foundation for Statistical Computing, Vienna, Austria. https://www.R-project.org/.

54. Rhodes, G., \& Jeffery, L. (2006). Adaptive norm-based coding of facial identity. Vision Res, 46(18), 2977-2987. doi:10.1016/j.visres.2006.03.002

55. Rhodes, G., Jeffery, L., Clifford, C. W., \& Leopold, D. A. (2007). The timecourse of higher-level face aftereffects. Vision Research, 47(17), 2291-2296.

56. Rhodes, G., Jeffery, L., Watson, T. L., Clifford, C. W., \& Nakayama, K. (2003). Fitting the mind to the world: face adaptation and attractiveness aftereffects. Psychol Sci, 14(6), 558-566.

57. Rouder, J.N., Morey, R. D., Speckman, P. L., \& Province, J. M. (2012). Default Bayes factors for ANOVA designs. Journal of Mathematical Psychology, 56, 356-374.

58. Rouder, J. N., Speckman, P. L., Sun, D., Morey, R. D., \& Iverson, G. (2009). Bayesian t tests for accepting and rejecting the null hypothesis. Psychonomic Bulletin \& Review, 16, 225-237.

59. Rosenfeld, A., Hummel, R. A., \& Zucker, S. W. (1976). Scene labeling by relaxation operations. IEEE Transactions on Systems, Man, and Cybernetics, (6), 420-433.

60. Sou, K. L., \& Xu, H. (2019). Brief facial emotion aftereffect occurs earlier for angry than happy adaptation. Vision research, 162, 35-42

61. Tiddeman, B., Burt, D.M., \& Perrett, D. (2001). Computer Graphics in Facial Perception Research, IEEE Computer Graphics and Applications, 21(5), 42-50.

62. Valentine, T., Darling, S., \& Donnelly, M. (2004). Why are average faces attractive? The effect of view and averageness on the attractiveness of female faces. Psychonomic Bulletin \& Review, 11(3), 482-487.

63. Webster, M. A., Kaping, D., Mizokami, Y., \& Duhamel, P. (2004). Adaptation to natural facial categories. Nature, 428(6982), 557-561. doi:10.1038/nature02420

64. Webster, M. A., \& MacLeod, D. I. (2011). Visual adaptation and face perception. Philos Trans $R$ Soc Lond B Biol Sci, 366(1571), 1702-1725. doi:10.1098/rstb.2010.0360 
914

915

916

917

918

919

920

921

922

923

924

925

926

927

928

929

930

931

932

933

934

935

936

937

938

939

940

941

942

943

944

945

946

65. Whitney, D., \& Levi, D. M. (2011). Visual crowding: a fundamental limit on conscious perception and object recognition. Trends Cognitve Science, 15(4), 160-168. doi:10.1016/j.tics.2011.02.005

66. Whitney, D., \& Yamanashi Leib, A. (2017). Ensemble Perception. Annu Rev Psychol. doi:10.1146/annurev-psych-010416-044232

67. Willenbockel, V., Sadr, J., Fiset, D., Horne, G. O., Gosselin, F., \& Tanaka, J. W. (2010). Controlling low-level image properties: The SHINE toolbox. Behavior Research Methods, 42(3), 671-684. doi:10.3758/Brm.42.3.671

68. Wolfe, B. A., Kosovicheva, A. A., Leib, A. Y., Wood, K., \& Whitney, D. (2015). Foveal input is not required for perception of crowd facial expression. Jouranl of Vission, 15(4), 11. doi:10.1167/15.4.11

69. Xu, H., Dayan, P., Lipkin, R. M., \& Qian, N. (2008). Adaptation across the cortical hierarchy: Low-level curve adaptation affects high-level facial-expression judgments. Journal of Neuroscience, 28(13), 3374-3383.

70. Yap, W.J., Chan, E., \& Christopoulos, G.I. (July 2016). Nanyang Facial Emotional Expression [N-FEE] Database - Development and Validation. Poster presented at the 23rd Congress of the International Association for Cross-Cultural Psychology, Nagoya, Japan.

71. Ying, H., \& Xu, H. (2017). Adaptation reveals that facial expression averaging occurs during rapid serial presentation. Journal of Vision, 17(1), 15. doi:10.1167/17.1.15

72. Ying, H., Burns, E. J., Lin, X., \& Xu, H. (2019). Ensemble statistics shape face adaptation and the cheerleader effect. Journal of Experimental Psychology: General, $148(3), 421$.

73. Young, A. W., \& Bruce, V. (2011). Understanding person perception. British Journal of Psychology, 102, 959-974. doi:10.1111/j.2044-8295.2011.02045.x

74. Zhao, L., \& Chubb, C. (2001). The size-tuning of the face-distortion after-effect. Vision Research, 41(23), 2979-2994. doi:Doi 10.1016/S0042-6989(01)00202-4

75. Zhang, G. L., Li, A. S., Miao, C. G., He, X., Zhang, M., \& Zhang, Y. (2018). A consumergrade LCD monitor for precise visual stimulation. Behavior research methods, 50(4), 1496-1502.

76. Zhao, Y., Zhen, Z., Liu, X., Song, Y., \& Liu, J. (2017). The neural network for face recognition: Insights from an fMRI study on developmental prosopagnosia. Neuroimage, 169, 151-161. doi:10.1016/j.neuroimage.2017.12.023 


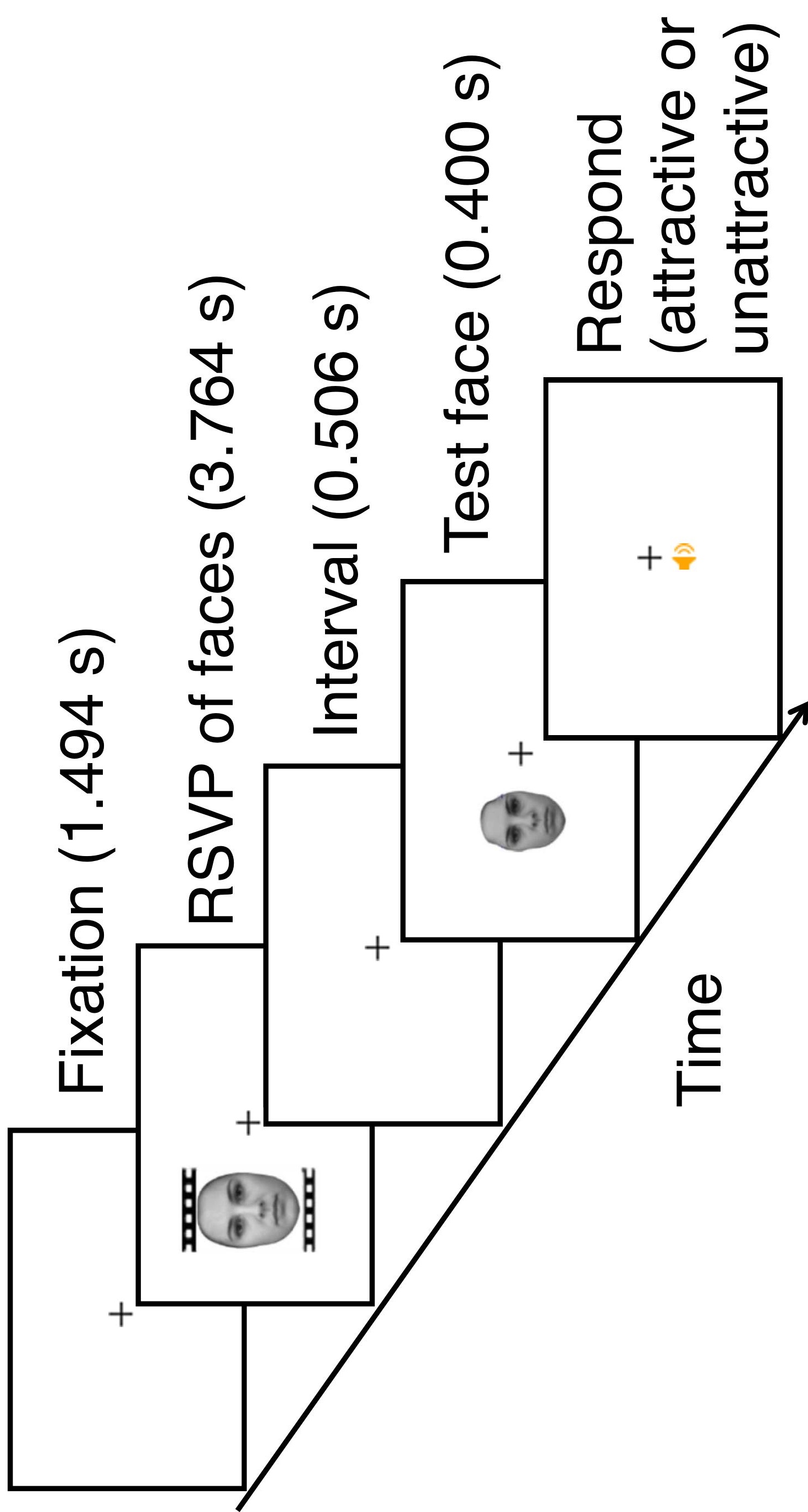




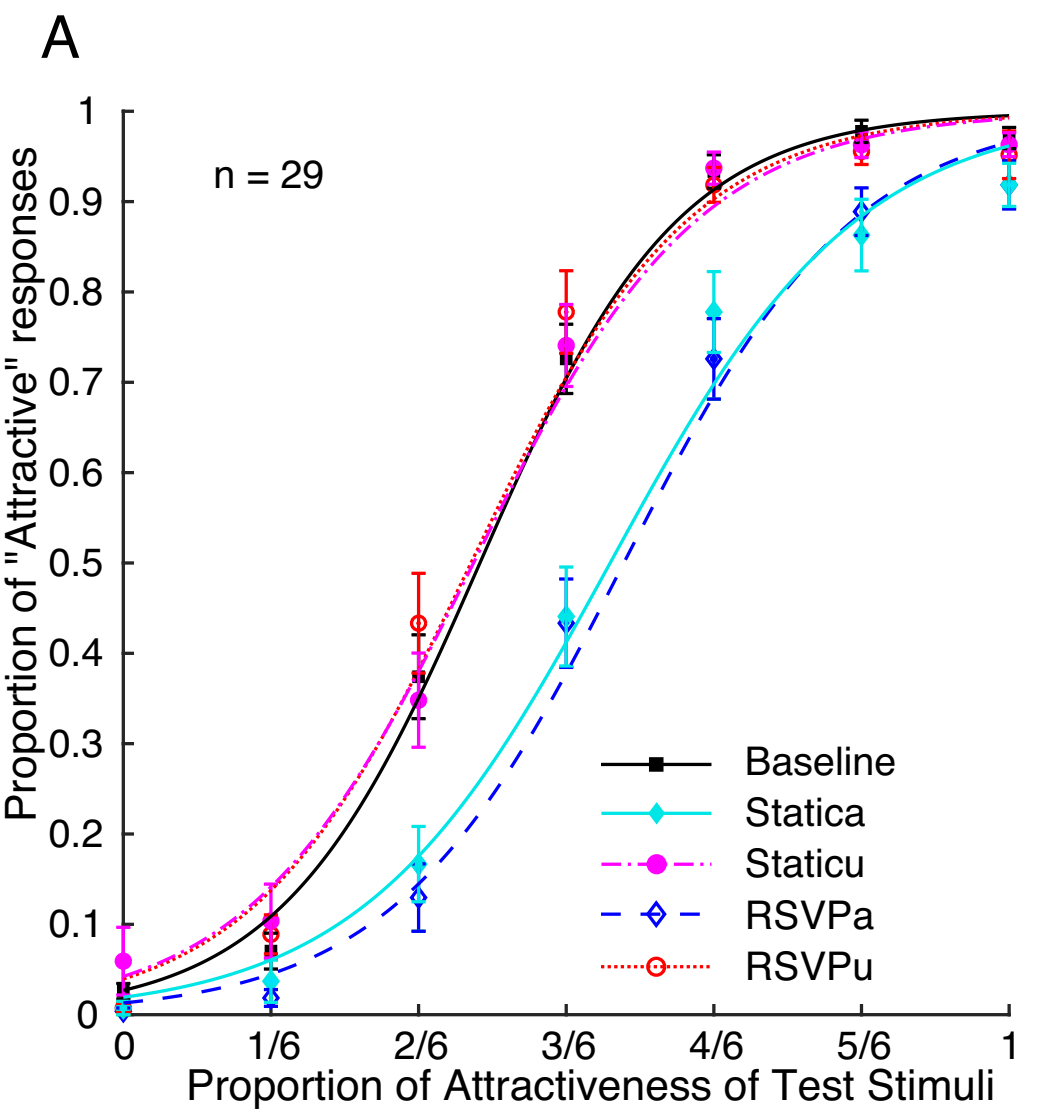

B
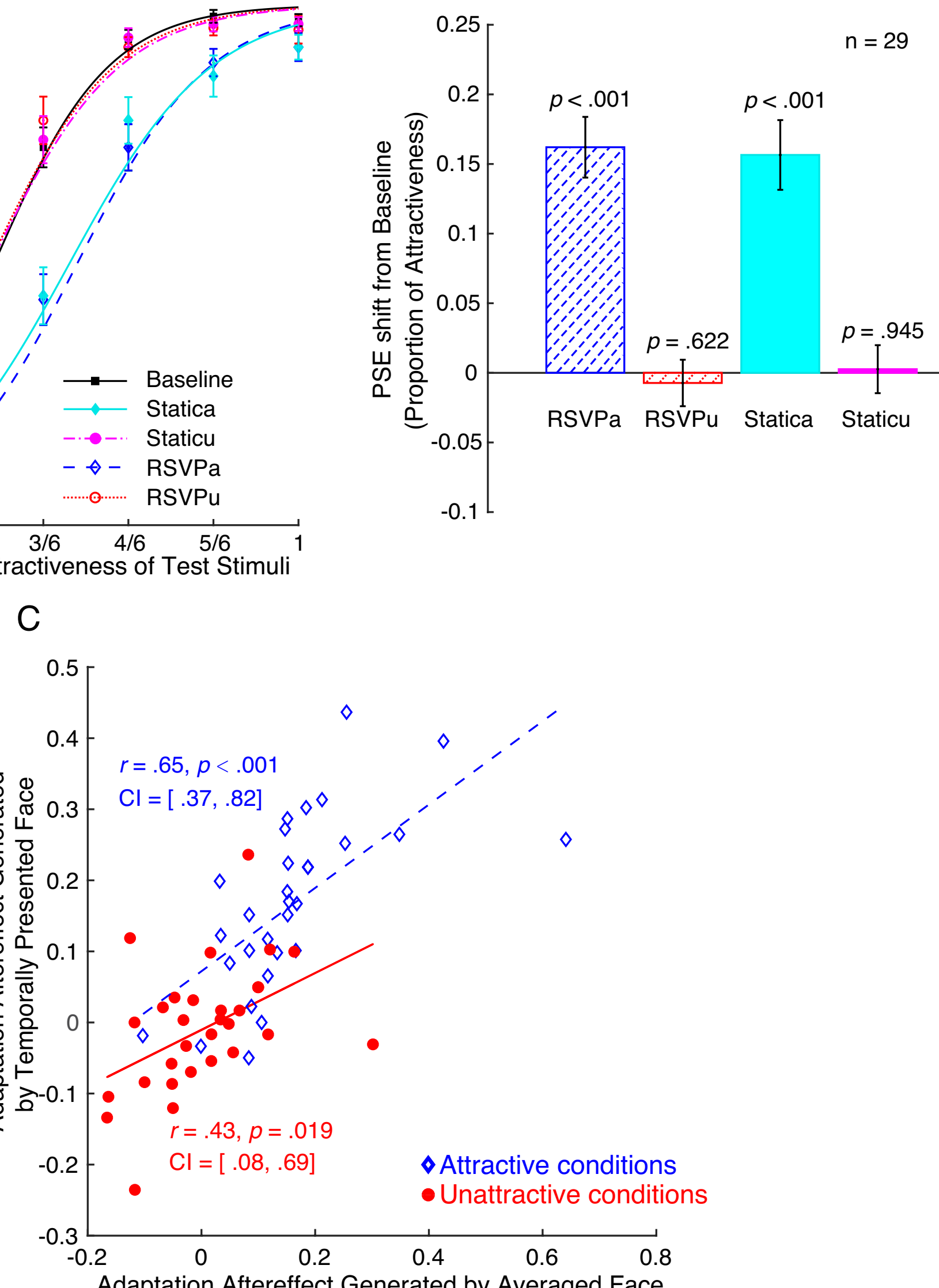


\section{Aigure 3}

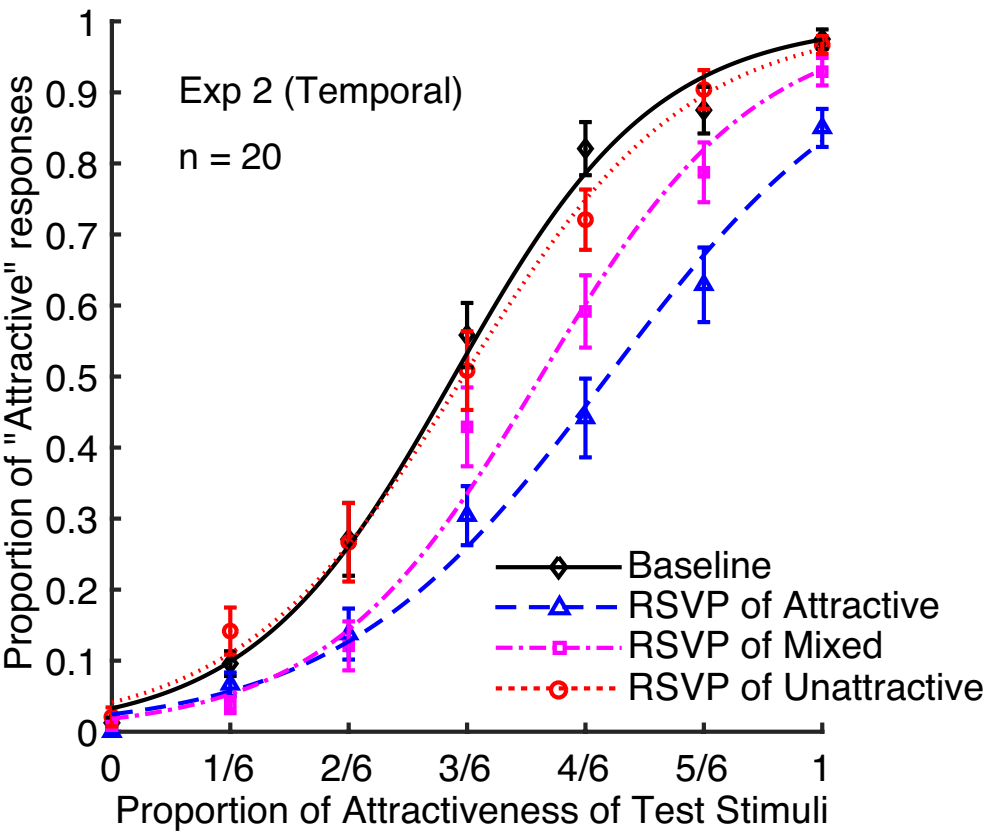

B

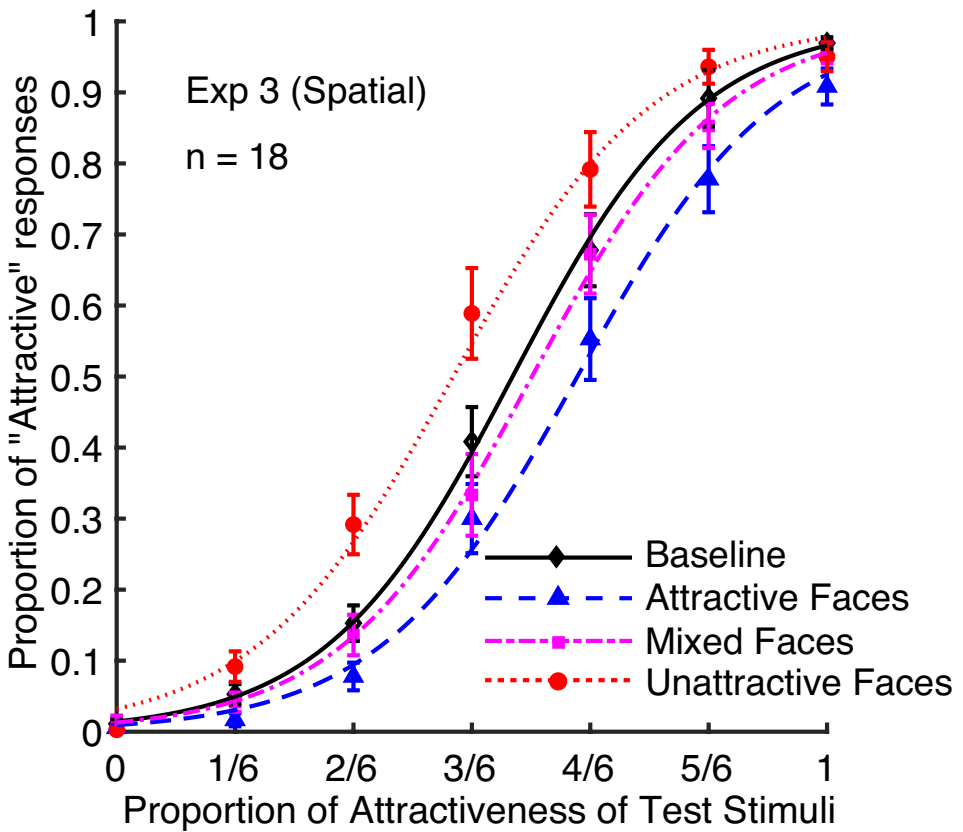

C

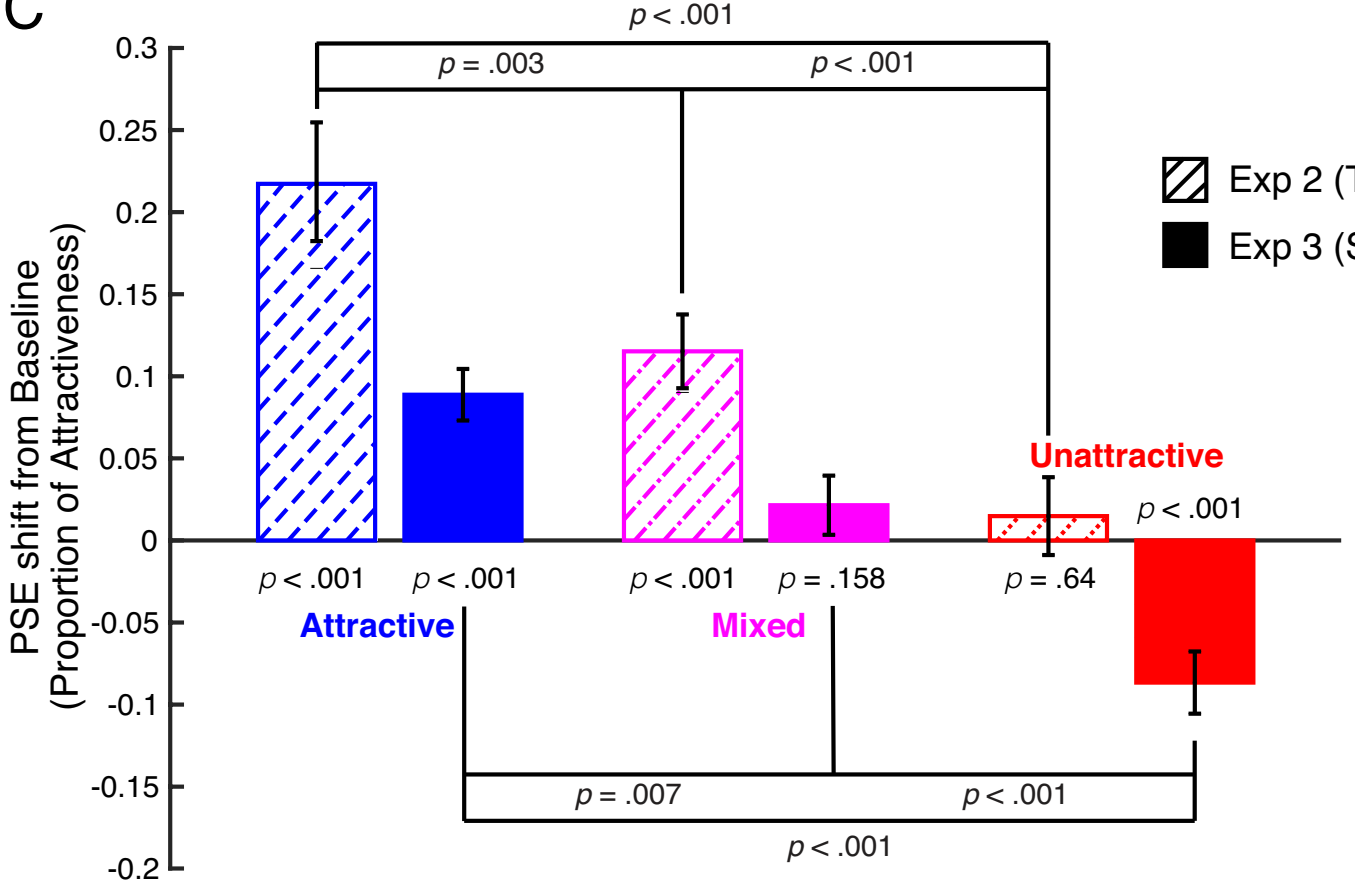

D

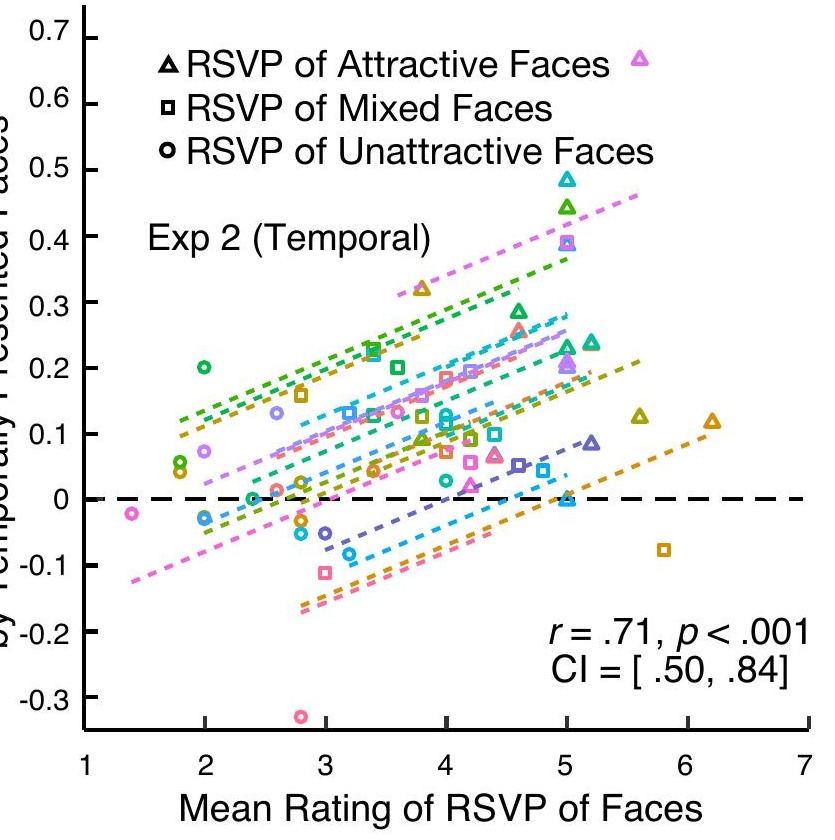

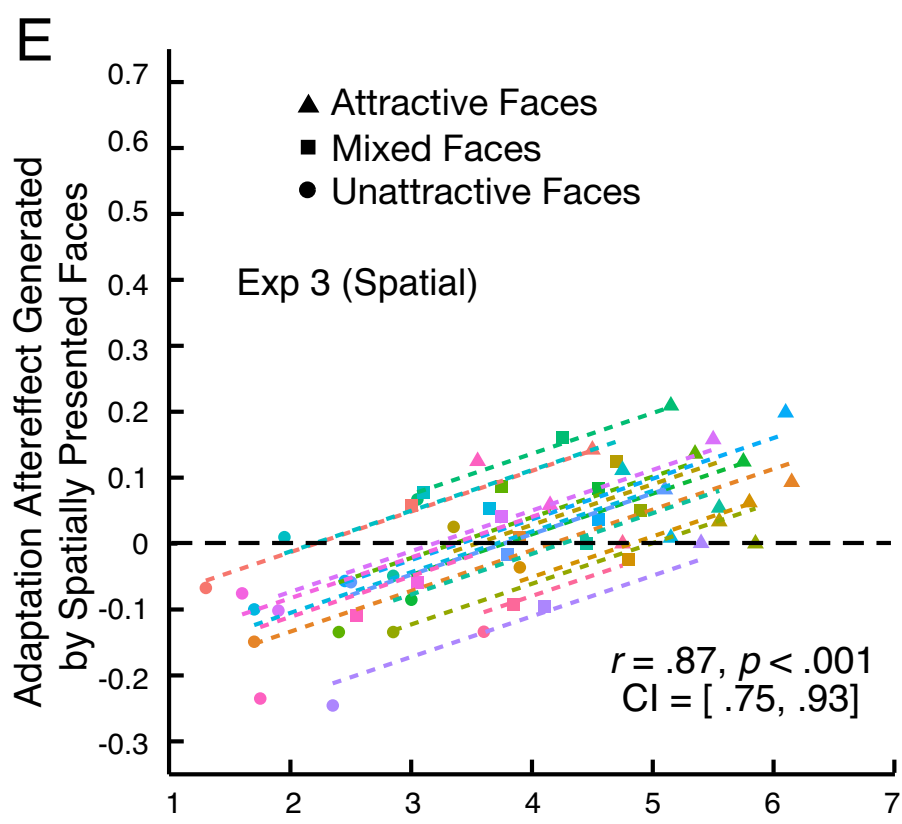

Gist Average of Spatially Presented Faces 


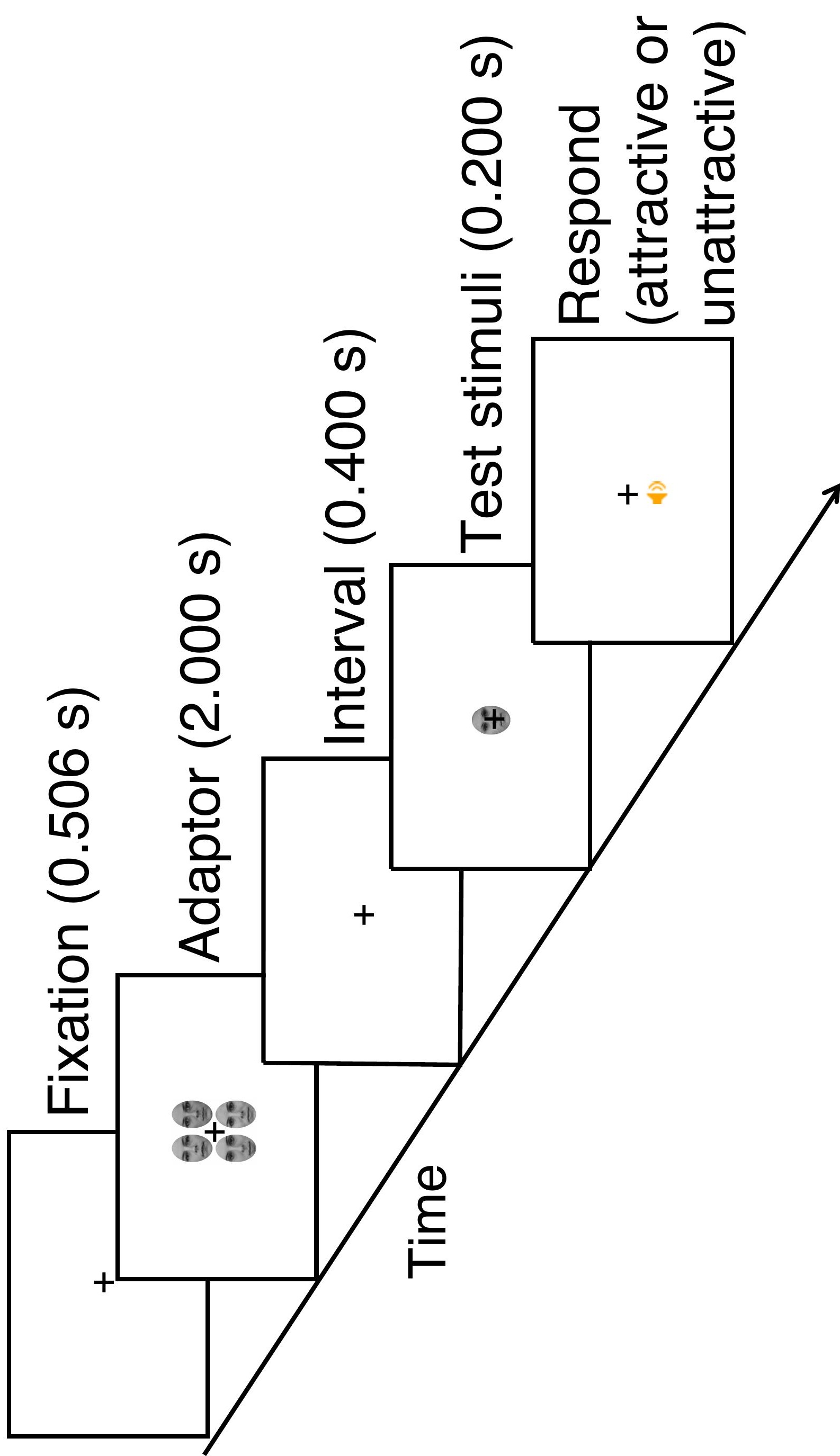


Figure 5
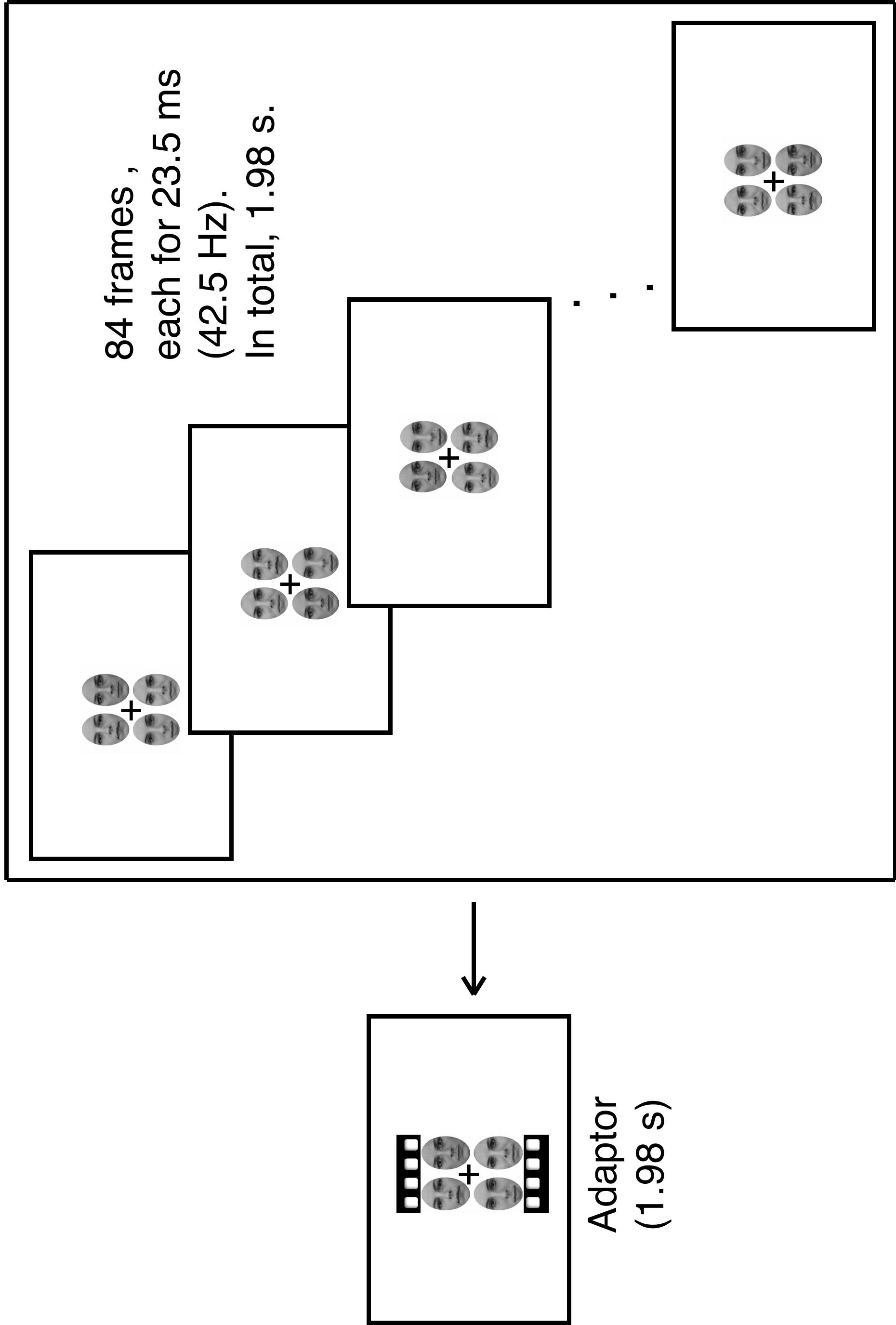
$A$

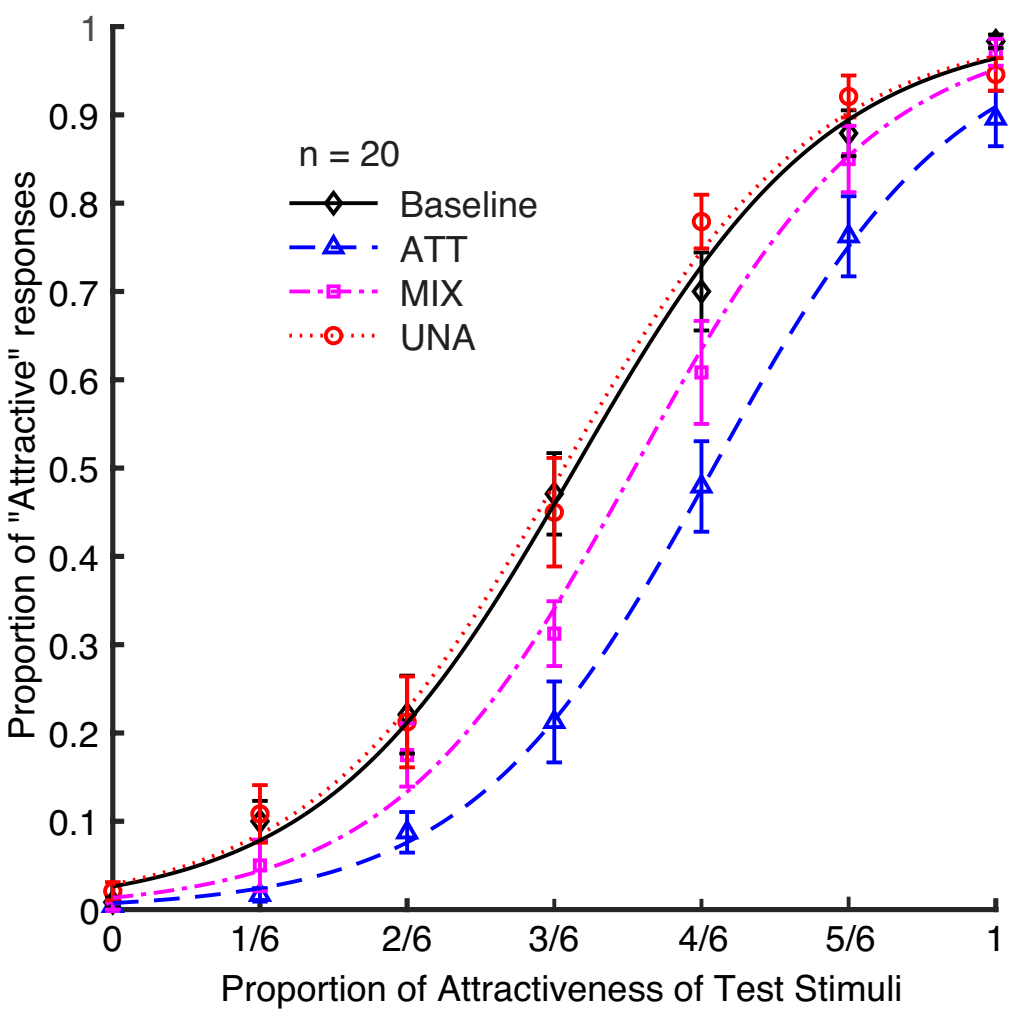

Proportion of Attractiveness of Test Stimuli
B

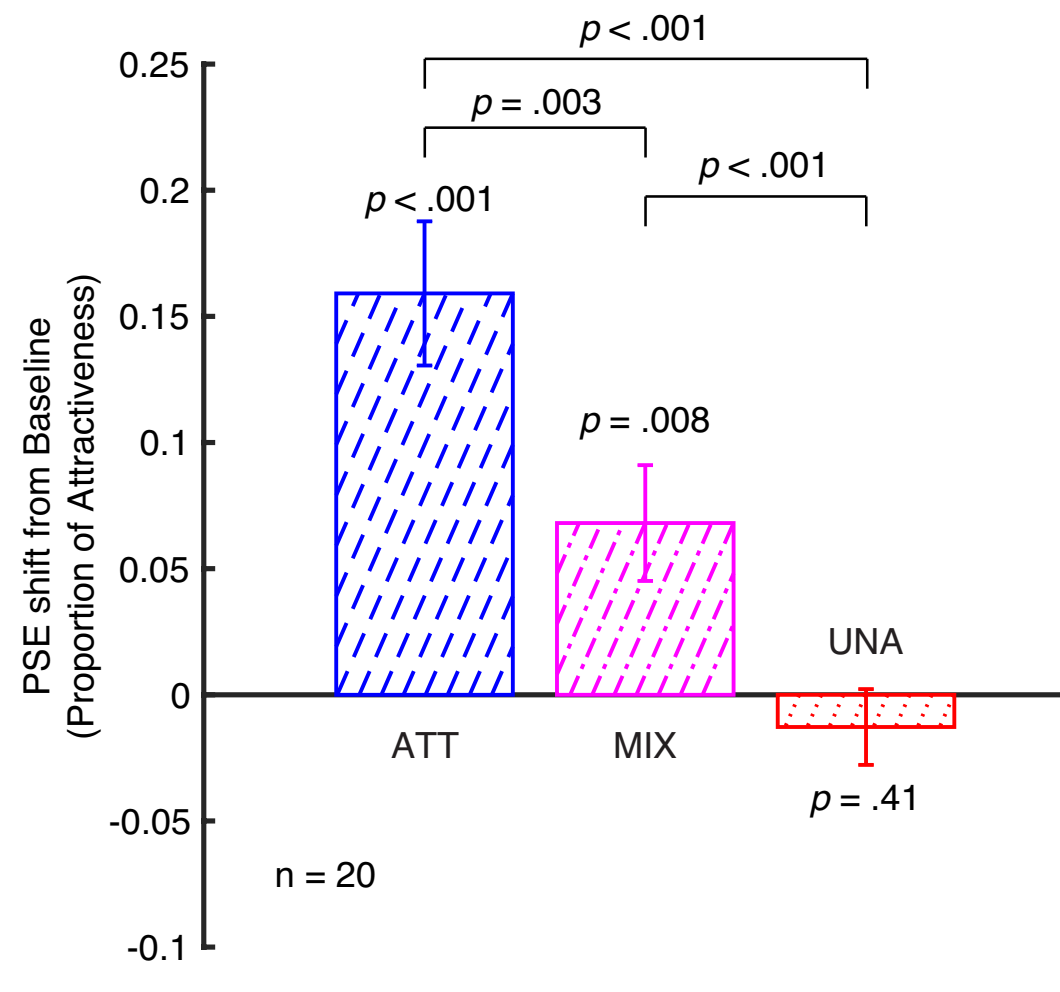

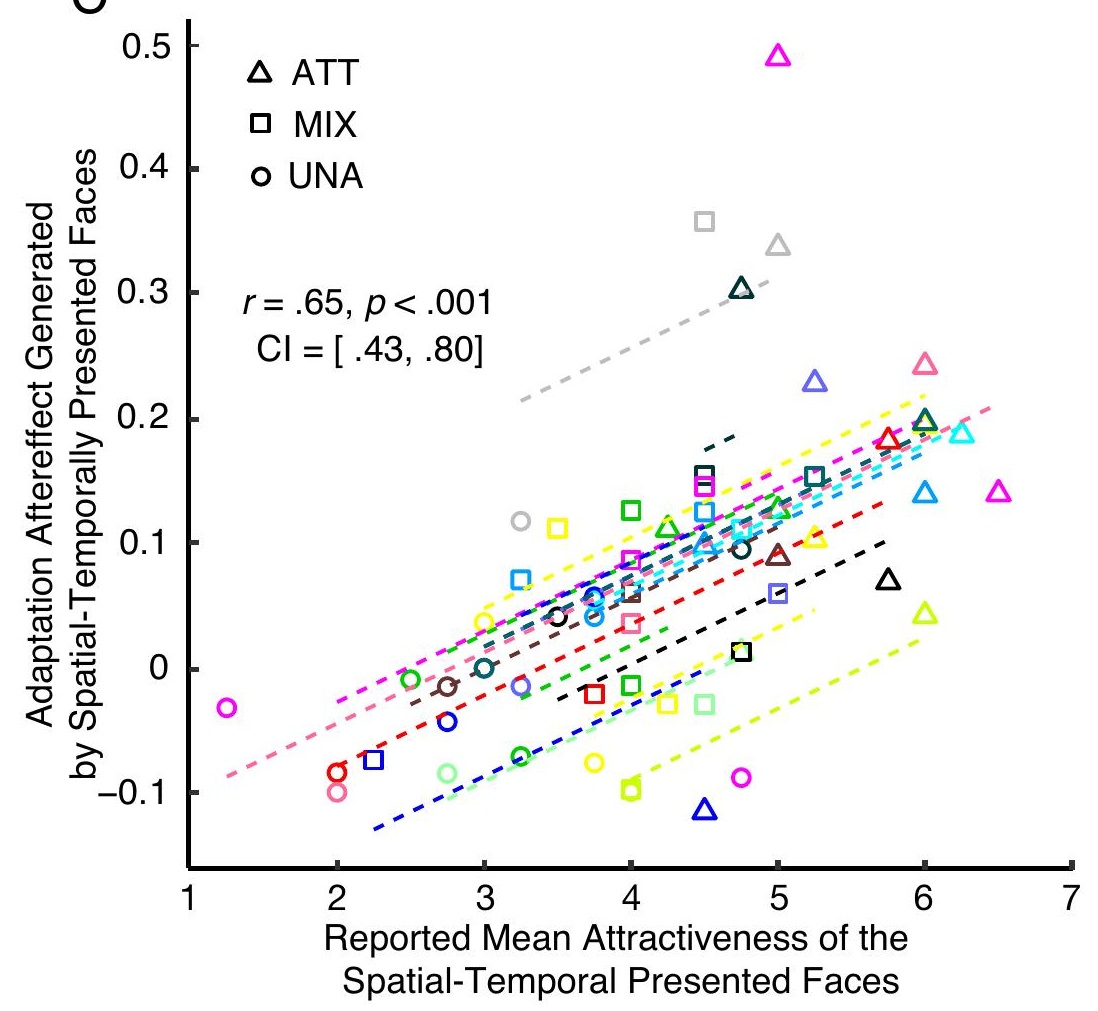




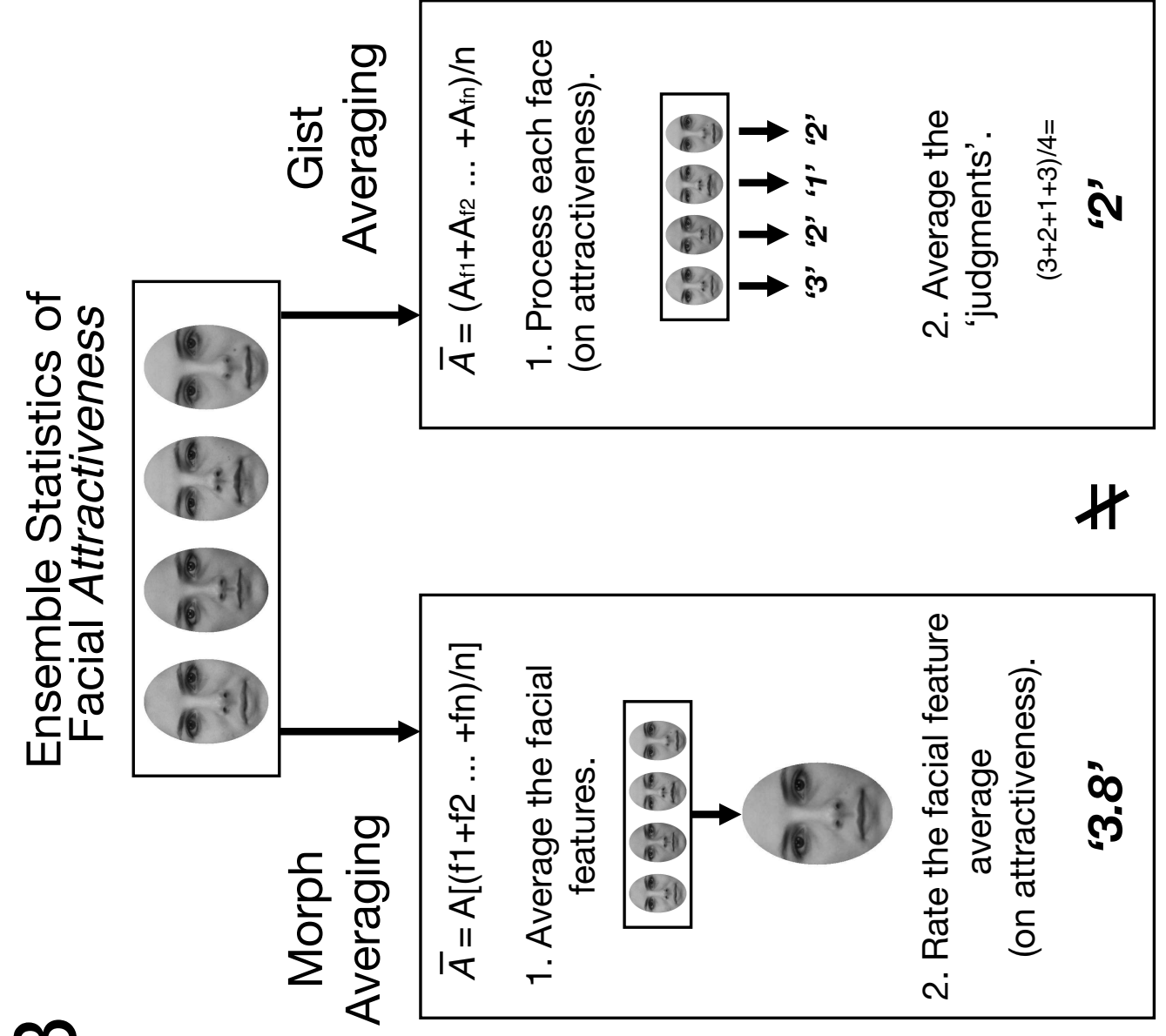

$m$

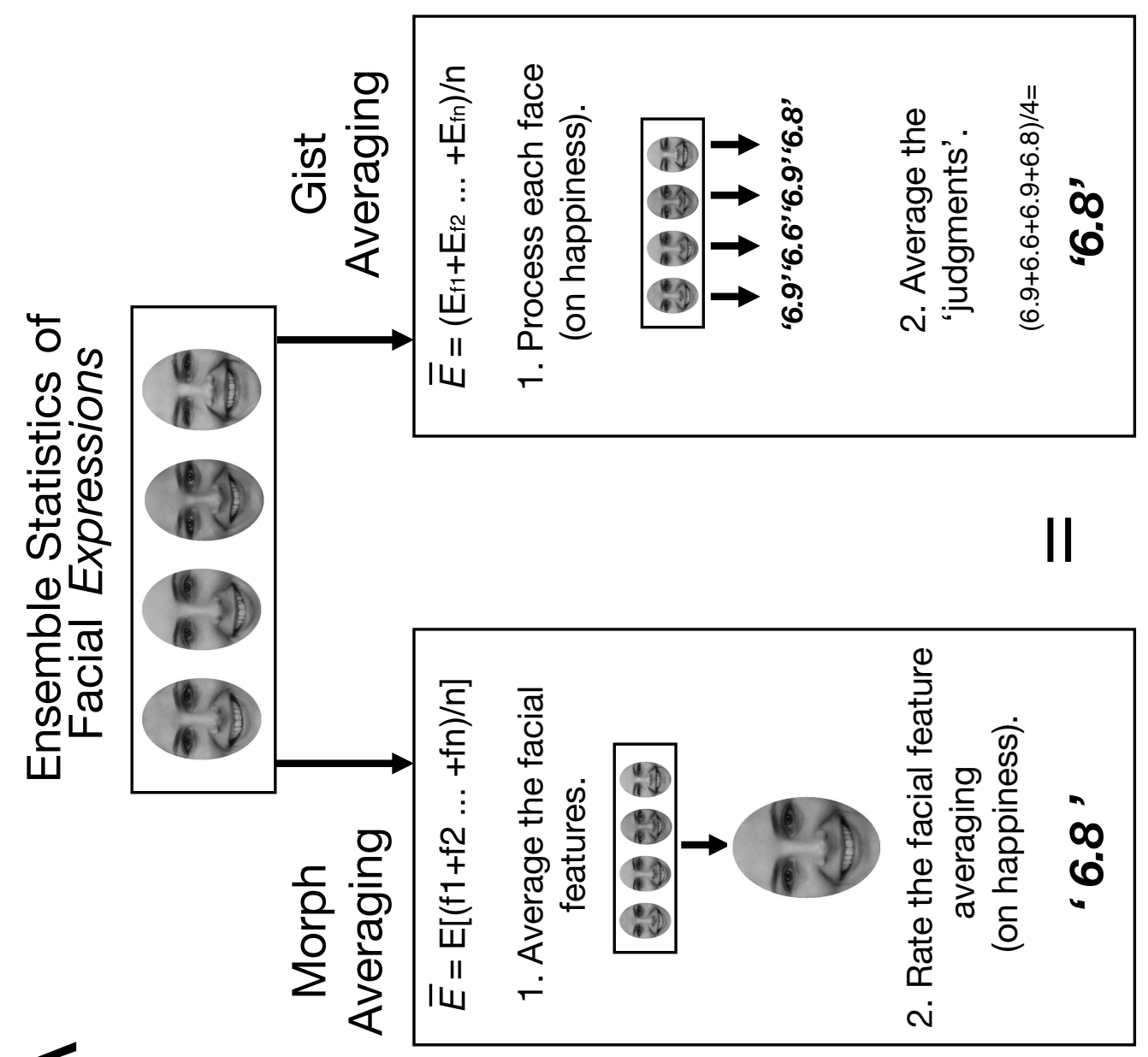

